\title{
In situ Alphavirus Assembly and Budding Mechanism Revealed by Cellular CryoET
}

David Chmielewski ${ }^{1}$, Michael F. Schmid ${ }^{2}$, Graham Simmons ${ }^{3}$, Jing Jin ${ }^{2,3 \#}$, Wah Chiu ${ }^{1,2,4 \#}$

\author{
Affiliations \\ ${ }^{1}$ Graduate Program in Biophysics, Stanford University, Stanford, CA 94305, USA \\ 2 Division of CryoEM and Bioimaging, SSRL, SLAC National Accelerator Laboratory, \\ Menlo Park, CA 94025, USA \\ ${ }^{3}$ Vitalant Research Institute, San Francisco, CA 94118, USA \\ ${ }^{4}$ Department of Bioengineering and James H. Clark Center, Stanford University, \\ Stanford, CA 94305, USA
}

\#Correspondence: wahc@stanford.edu and JJin@vitalant.org

\section{Summary}

Chikungunya virus (CHIKV) is an alphavirus and the etiological agent for debilitating arthritogenic disease in humans. Previous studies with purified virions or budding mutants have not resolved the structural mechanism of alphavirus assembly in situ. Here we used cryogenic electron tomography (cryoET) imaging of CHIKV-infected human cells and subvolume classification to resolve distinct assembly intermediate conformations. These structures revealed that particle formation is driven by the spike envelope layer. Additionally, we showed that asymmetric immature nucleocapsids (NCs) provide scaffolds to trigger assembly of the icosahedral spike lattice, which progressively transforms immature NCs into icosahedral cores during virus budding. Further, cryoET of the infected cells treated with neutralizing antibodies (NAbs) showed that NAb-induced blockage of CHIKV assembly was achieved by preventing spike-spike lateral interactions that are required to bend the plasma membrane around NC cores. 
bioRxiv preprint doi: https://doi.org/10.1101/2021.10.14.464449; this version posted October 14,2021 . The copyright holder for this preprint

(which was not certified by peer review) is the author/funder, who has granted bioRxiv a license to display the preprint in perpetuity. It is made available under aCC-BY-NC-ND 4.0 International license.

These findings provide molecular mechanisms for designing antivirals targeting spikedriven assembly/budding of viruses.

\section{Keywords}

Chikungunya virus, alphavirus, virus assembly and budding, enveloped virus, cryogenic electron tomography, subtomogram averaging, antibody 


\section{Introduction}

2 Chikungunya virus (CHIKV) is the most common alphavirus infecting humans worldwide, causing epidemics in all continents except Oceania and Antarctica.

$4 \quad$ Transmitted primarily by Aedes mosquitoes, CHIKV infection is associated with severe

5 symptoms of debilitating and often chronic polyarthritis in infected individuals (Silva and Dermody, 2017). No licensed vaccine or antivirals are available for CHIKV treatment. The CHIKV virion is $\sim 70 \mathrm{~nm}$ in diameter, with a membrane-embedded envelope glycoprotein (GP) shell of 240 copies of E1-E2.E3 heterodimers arranged as 80 trimeric spikes, and an inner nucleocapsid (NC) core of 240 capsid proteins (Cps) that encapsulate the $11.5 \mathrm{~kb}$ plus-sense (+) genomic RNA (gRNA) (Sun et al., 2013). In CHIKV-infected cells, viral structural proteins (Cp, E1, E2, E3) are synthesized as a single polyprotein precursor molecule. Cp auto-proteolytically cleaves itself from the polyprotein and oligomerizes into nucleocapsid-like-particles (NLPs) in the cytosol, mediated by $\mathrm{Cp}$ interactions with the negative-charged gRNA and $\mathrm{Cp}-\mathrm{Cp}$ interactions (Choi et al., 1991, 1997; Nicola et al., 1999). The remaining polyprotein is inserted into the ER, processed into E1/E2.E3 heterodimers and trafficked through the secretory pathway to the plasma membrane (PM) as trimeric spikes. E3 is cleaved off by host furin and furin-like proteases and stays associated with the nascent virions (Basore et al., 2019; Zhang et al., 2011). Convergence of cytosolic NLPs and membraneembedded spikes at the cell surface results in assembly and budding of enveloped virions with icosahedral spike and NC layers (Cheng et al., 1995).

Formation of enveloped virus particles requires viral protein and/or host factor-induced curving of a cellular membrane around viral cores followed by membrane scission. Some viruses such as retroviruses, rhabdovirus and filoviruses, recruit host ESCRT machinery to drive virus assembly/budding and their NC cores alone can bud as virus-

27 like particles (VLPs) without viral glycoproteins embedded in the lipid envelope. In contrast, production of CHIKV VLPs requires co-expression of GPs and Cp, while budding is reported to be ESCRT-independent (Noranate et al., 2014; Taylor et al., 30 2007). Numerous studies suggest that alphavirus budding is mediated by both vertical spike:Cp interactions and lateral interactions between spikes (Forsell et al., 2000; 
32 Suomalainen et al., 1992). Insertion of the intracellular tail of E2 into the hydrophobic

33 pocket of Cp C-terminal domain mediates the vertical Spike-Cp interactions, while

34 lateral E1 self-interactions form the surface envelope lattice (Cheng et al., 1995; Tang et

35 al., 2011; Zhang et al., 2011).

Two distinct models for the major driving force in budding have been proposed: spikeNC binding and spike-spike shell interactions (Garoff et al., 2004). Purified Cps assemble in vitro into core-like-particles (CLPs) with fragile, imperfect icosahedral symmetry (Mukhopadhyay et al., 2002; Wang et al., 2015). Microinjection of in vitroassembled CLPs into spike-expressing cells induces low levels of virus budding, suggesting interactions between spikes and preassembled cytosolic NLPs drive budding (Cheng and Mukhopadhyay, 2011; Snyder et al., 2011). In support of the alternative spike-driven model, $\mathrm{Cp}$ mutants deficient in $\mathrm{Cp}-\mathrm{Cp}$ interactions can still form virions with viral spikes, though at a lower efficiency than the wild-type (wt) (Forsell et al., 1996). This suggests that spike-spike interactions and spike-Cp interactions are sufficient to drive the assembly and budding of icosahedral particles without a preassembled NC. There are also reports of propagation of capsidless alphavirus via infectious microvesicles at a titer logs lower than the wt virus (Ruiz-Guillen et al., 2016). How spikes mediate packaging of gRNA in membrane vesicles is largely unknown. It is unclear whether any of these budding models, derived from viral mutants, are applicable to the case of wt alphavirus budding in situ.

Our previous study demonstrated that neutralizing antibodies (NAbs) inhibit CHIKV budding by crosslinking spikes at the outer surface of the PM and trapping NLPs inside CHIKV-infected cells (Jin et al., 2015, 2018). It suggests that spike organization on the infected cell surface is critical for virion assembly and budding. What is left unaddressed in that study are the molecular details of NAb-bound spike organization that inhibit membrane bending. Defining the difference between the spike organization in the normal virus budding process and that of budding inhibitory spike-NAb complexes can

61 inspire future therapeutic antiviral strategies. In the same study, we reported lack of icosahedral symmetry in budding-arrested NLPs and suggested it could either be a 
63 structural feature of immature cytosolic NLPs or a result of disassembly of budding-

64 arrested NLPs. NC assembly is a potential antiviral target and necessitates further

65 characterization of NC morphogenesis prior-to and during budding (Wan et al., 2020).

66 Recently, Cp-gRNA interactions were found to differ between cytosolic NLPs and virion-

67 NCs (Brown et al., 2020), supporting previous reports of physical differences between

68 the two populations and suggesting currently-uncharacterized morphological changes

69 occur during virus budding (Lamb et al., 2010).

71 To determine the assembly events leading to CHIKV mature particle formation, we

72 utilized cryogenic electron tomography (cryo-ET) imaging and subtomogram averaging

73 to reveal structures of intermediately assembled particles at multiple stages of virus

74 budding in CHIKV-infected human cells. Additionally, we resolved structures of self-

75 assembled spikes and NLPs in the absence of spike-Cp interactions. Based on these

76 structures, our study defines the mechanistic roles of spikes and immature NCs in

77 CHIKV budding and elucidates conformational changes of NC during virion assembly. In

78 addition, we present a method of classifying snapshots of the alphavirus budding

79 process into discrete ensemble averages of conformational states in cellulo.

\section{Results}

82 CryoET and subtomogram classification of CHIKV budding intermediates in situ

83 CHIKV particle assembly and budding, driven by interactions between NCs and

84 membrane-embedded spikes, is known to occur at the PM of infected cells. To capture

85 the dynamic process in the near-native state, we imaged U2OS cells, a human bone

86 osteosarcoma cell line, that were infected with CHIKV-181 vaccine strain and

87 embedded in vitreous ice. Collection of tomographic tilt series at the infected cell

88 peripheries 8 hours post-infection revealed a variety of budding phenotypes (movie S1),

89 with CHIKV assembly events located at the PM of the cell body (Fig. 1A-D), on long

90 intercellular extensions (>10 $\mu \mathrm{m}$ ), short extensions (typically 2-10 $\mu \mathrm{m}$ in length), and thin

91 branching extensions composed solely of viral particles (Fig. 1A-B). Particles at various

92 intermediate stages of budding at the PM, as well as fully assembled virions released

93 into the extracellular space, were observed (Fig. 1E-F), thus capturing snapshots of the 
94 entire CHIKV assembly/budding process. The high concentration of CHIKV budding at

95 specific regions of the cell periphery and variety of observed cell extensions is consistent with previous reports of highly localized budding and virus-induced branching

97 structures at the cell periphery from 2-D electron microscopy images of plastic-

98 embedded material (Birdwell et al., 1973; Laakkonen et al., 1998; Martinez et al., 2014;

99 Pavan et al., 1987). Interestingly, CHIKV replication spherules, where viral RNAs are synthesized, were occasionally observed near cytosolic NLPs and budding viruses (Fig. 1C-D, Fig. S1). It is conceivable that viral RNAs are synthesized and immediately packaged into NLPs that bud into virions, all near the PM.
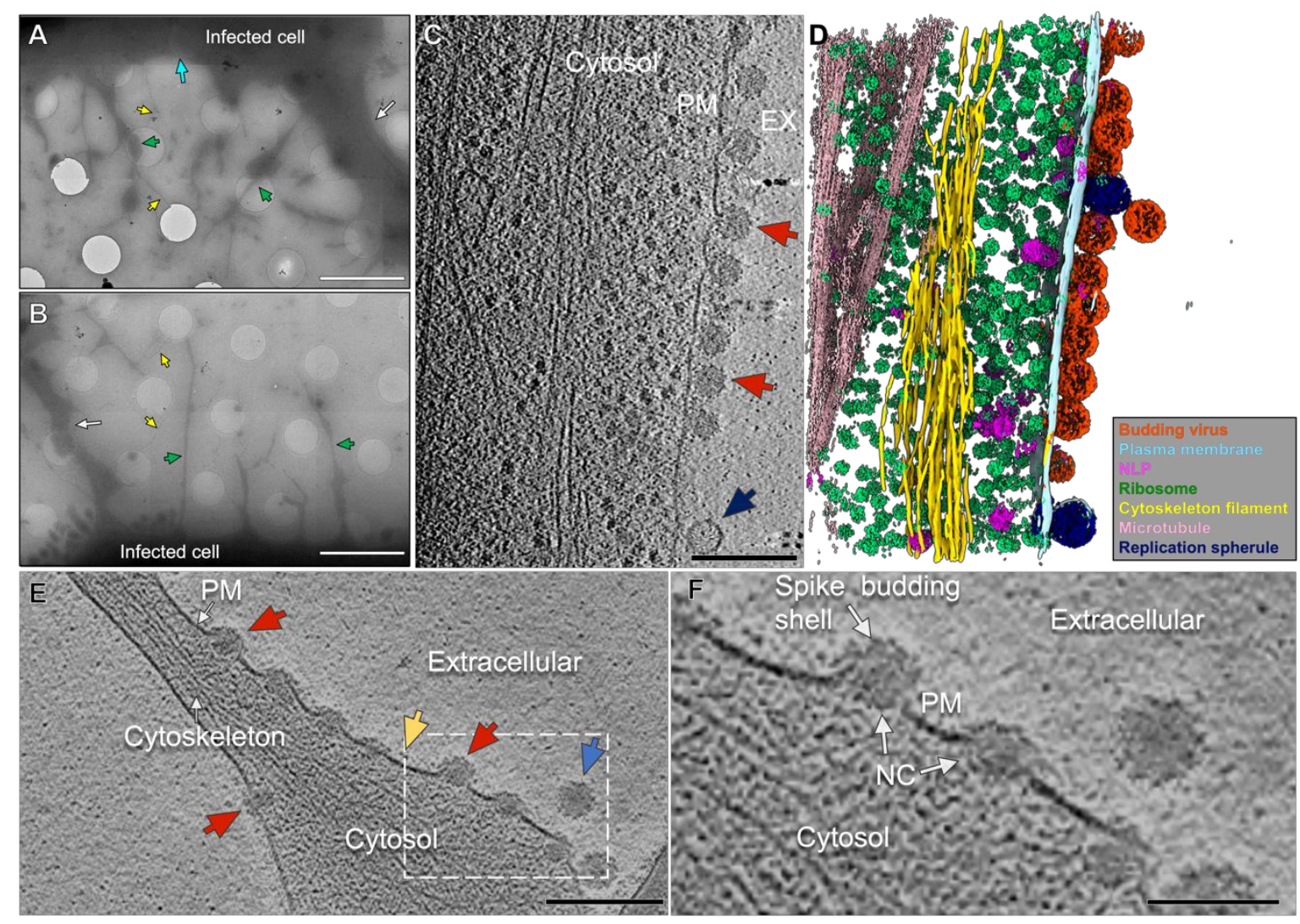

Figure 1. CHIKV assembly and budding at the infected-cell periphery.

(A-B) Low magnification images of the cell periphery reveal cell body (cyan arrow), long intercellular extension (white arrow), short extensions enriched in virus assembly features (green arrows), and thin extensions of viral particles (yellow arrows) emanating from the short extensions or cell body. Scale bars $5 \mu \mathrm{m}$. (C) Tomographic slice of cell periphery depicting virus 
111 budding events (red arrows) and RNA replication spherules (navy blue arrow) at the PM with (D)

112 corresponding 3D segmentation of cellular features. (EX: extracellular space) Scale bar $200 \mathrm{~nm}$.

113 (see also Fig. S1) (E) Tomogram slice of short extension with budding intermediate particles

114 (red arrow), spikes (yellow arrow), and cell free virion (blue arrow). Scale bar $200 \mathrm{~nm}$. (F)

115 Enlarged view of the boxed region in (E) shows intermediate viral assembly complexes at the

116 PM, composed of a spike budding shell and nucleocapsid (NC). Scale bar $100 \mathrm{~nm}$.

On the virus-infected cell periphery we frequently identified thin extensions formed by incomplete particles, often the width of just a single virion $(\sim 70 \mathrm{~nm}$ diameter, $<5 \mu \mathrm{m}$ length) and lacking bundled cytoskeleton filaments (Fig. S2). Thin extensions possess a diversity of particle structures, with differences in the levels of completion and structural conformations (Fig. S2). Convergence of two opposing membranes containing nearcontinuous budding intermediates was observed to give rise to strings of incomplete particles with a continuous membrane connection. Due to the lack of sufficient spikes to finish enclosing the NC as an icosahedron, these linked particles are unlikely to complete the assembly of full virions and therefore were excluded from the following analysis.

In order to determine in situ conformations of the CHIKV particle assembly process, we extracted 1,918 budding intermediate particles at the cell periphery (Fig. 2C, 3A). To address conformational and compositional heterogeneity within snapshots of the budding process, individual particle subvolumes were discretized into 3D classes

133 through an unbiased and iterative multi-reference classification based on structural

134 similarity using C5 symmetry (Fig. S3A, see "Methods" section). This resulted in 12 3D

135 reconstructions of discrete virus budding conformations (Fig. 2) that displayed

136 progressive levels of budding, with different extents of completion of the spherical

137 budding shell. The 3D class averages 4, 6, 9 and 10 possessed weak density of the PM

138 and trailing end of NC that became blurred and masked-out during refinement. This is 139 likely due to heterogeneity among individual particles within those classes and/or lack of 140 symmetry of these regions relative to the leading end of budding particles. 
142 To analyze progression of the CHIKV budding pathway, the population of particles

143 within each 3D class was then analyzed in relation to budding level (i.e., percentage

144 completion of the budding shell). Budding level was determined by using the mature

145 icosahedral virion as a reference and counting the number of spikes (out of 80 ) covering

146 the budding shell of each low-resolution intermediate class average (Fig. 2D). From a

147 total of 1,375 budding intermediate particles within the 12 classes that converged to

148 interpretable 3D structures, 288 particles (21\%) were classified into structures

149 containing 10-20 spikes (classes 1-3,12-25\% complete), 191 particles (14\%) were

150 classified into structures containing 40-70 spikes (classes 4-8, 50-88\% complete), and

151868 particles (63\%) were classified into structures containing 75-80 spikes (classes 9-

152 12, 94-100\% complete). The low particle numbers and comparatively low resolution

153 classes in the $50-88 \%$ of completed virion range (budding classes 4-8) suggest CHIKV

154 assembly progression at these mid stages is more transient than early (budding classes

155 1-3) and late stages (budding classes 9-12).
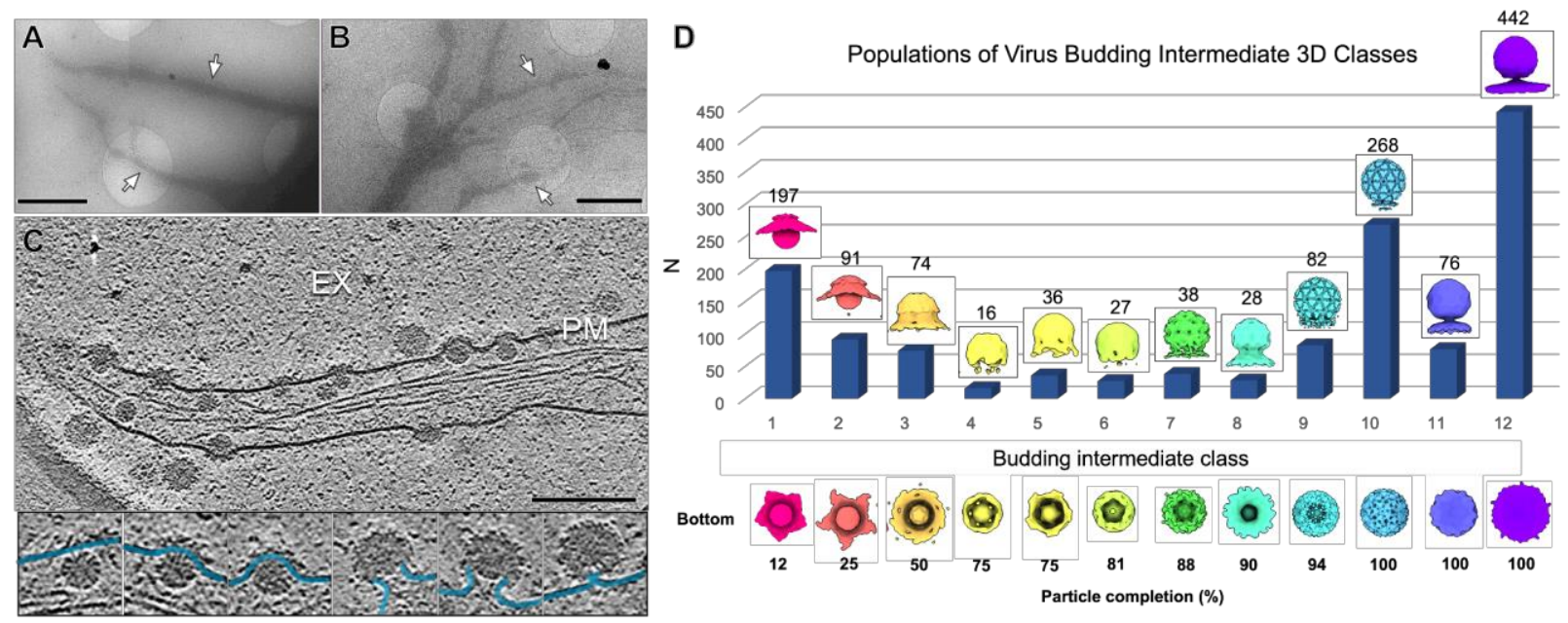

158 Figure 2. Classification and enumeration of CHIKV budding intermediates.

159 (A-B) Images of virus-infected cells with extensions enriched in virus assembly (white arrows).

160 Scale bars $2 \mu \mathrm{m}$. (see also Fig. S2) (C) Tomogram slice image depicts snapshots of the virus

161 budding process. Selected particle images (below) reveal heterogeneity based on

162 conformations of the bending PM (blue). (EX- extracellular space), Scale bar $200 \mathrm{~nm}$. (D)

163 CHIKV-budding intermediate 3D classes determined by subvolume classification. Density maps

164 of each class average (1-12) are colored uniquely and displayed with side-view and bottom-view 
165 (viewed from below the PM). The number of particles $(\mathrm{N})$ assigned to each class displayed as a bar graph with respective $\mathrm{N}$ listed above. (see also Fig. S3)

To enhance low-resolution image contrast for visualization of CHIKV particles, we performed another set of experiments which utilized a Volta phase plate (Danev et al., 2014). Previous structural studies of purified alphavirus particles have described significant inter- and intra-particle heterogeneity (Chen et al., 2018; Zhang et al., 2011) but concerns about the fragility of enveloped viruses to purification have cautioned conclusions about the relevance of structural heterogeneity to alphavirus assembly in situ. Therefore, our imaging of CHIKV budding and released virions in situ eliminates the need for in vitro purification and handling of virus particles prior to vitrification. Within released virions, relatively absent density was consistently observed at one side of the particle between spike and NC core layers (Fig. S4). Further, unidentified molecular complexes are observed at the base of the V-shaped viral envelope in the relatively absent density region of many released particles (Fig. S4D). Relatively absent density was also observed in released multi-core particles in the region between NCs, suggesting it arises from a lack of spike-NC contacts enclosing the icosahedron (Fig. S4D-E). Interestingly, the trailing-end of late-stage budding particles tethered to the PM displays similar relatively absent density and geometry of the viral envelope (Fig. S4B). Therefore, deviations from the icosahedral lattice within released virus particles appear to directly result from late events in virus budding from the host cell membrane.

\section{In situ structures of progressing CHIKV budding intermediates}

To further analyze structures of spike:immature NC complexes at the molecular level during particle assembly, we performed additional 3D refinement of released virions and four budding-intermediate class averages that displayed low-resolution icosahedral features (see Methods). Icosahedral (5-3-2) symmetry is a feature of purified CHIKV particles (Sun et al., 2013), but it was unknown if CHIKV assembly progressed through 193 partially-icosahedral intermediates or if full icosahedral symmetry of infectious particles arose from protein rearrangements following virus release. Particle subvolumes were aligned with C5 symmetry, resulting in the earliest budding structure displaying a 5-fold 
pentagon at the leading end of budding, while the other four structures displayed excellent pentagon and hexagon assemblies with 5-fold, 3-fold, and 2-fold symmetry axes (Fig. 3B). The five subvolume averages of discrete budding states range in resolution from $8.3 \AA$ (released) to $\sim 44 \AA$ ("docking") (0.143 FSC criterion) (Fig. 3B, Fig. $\mathrm{S} 3 \mathrm{~B})$. Subnanometer resolution in the released virion average is validated by the visualization of transmembrane helices of E1/E2 (Fig. S3C-D). The differences in resolution among the budding intermediate ensemble averages are likely caused by increased conformational flexibility in those assembly states with less icosahedral symmetry constraints and compositional differences between aligned particles with slightly different budding levels.

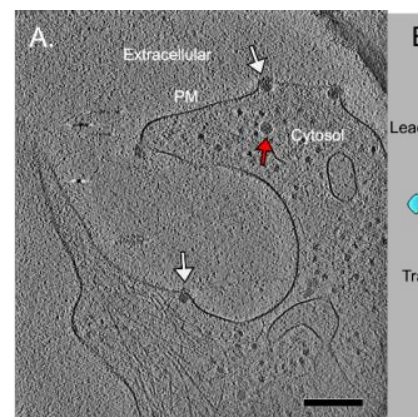
B. Budding intermediate averages

\section{Cytosolic NLPs} Top Side

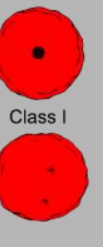

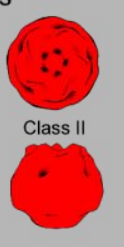
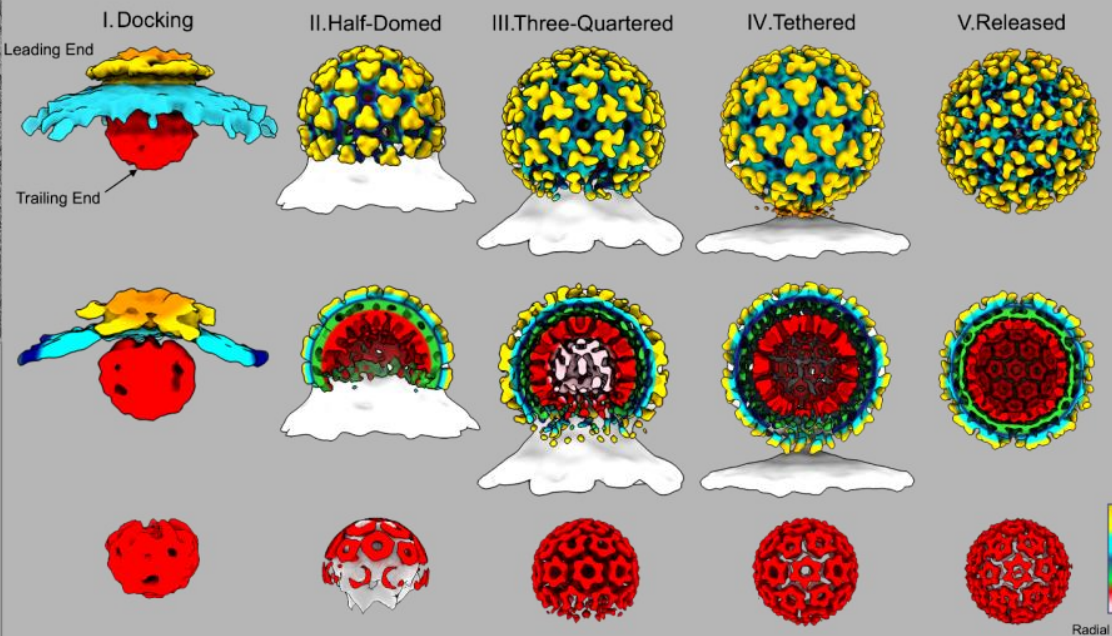

\section{Figure 3. Refined structures of CHIKV assembly intermediates.}

209 (A) Tomogram slice image displays budding intermediate particles at the PM (white arrows) and an apparently cytosolic NLP (red arrow). Scale bar $200 \mathrm{~nm}$. (B) Radially-colored density maps of five CHIKV ensemble subtomogram averages arranged in accordance with budding progression from earliest budding level ("I.Docking") to latest ("V.Released" virion) with half-cut representations (middle row) and NC-zoned densities (bottom row). PM and NC density ("HalfDomed", bottom row) from class averages prior to additional 3D refinement depicted as white surfaces. (see also Fig. S3 \& S4) (C) Subtomogram average structures of two cytosolic NLP classes. Class I displays no interpretable 5-fold symmetry while class II shows weak five-fold symmetry at one pole. (also see Fig. S4 \& S5). 
219 Within the budding intermediate averages, a striking correlation between icosahedrallysymmetric regions of the spike budding shells and their underlying NC core was observed at the "leading end" of budding (Fig. 3B). In contrast, there is a lack of detectable symmetry in the "trailing end" of each intermediate NC's structure, where no spikes are present (Fig. 3B, Fig. S4A-C). Even within the latest-stage budding conformation ("tethered"), weak density of the final penton of spikes at the trailing end is matched by a disordered pentamer of Cps in the NC below it (Fig. S4C). Based on these structures, as the icosahedral spike shell grows during budding, it appears to reorganize those Cps of the immature NC below into matching icosahedral symmetry

228 through 1:1 spike:Cp interactions. This result revealed that assembly progresses through partially icosahedral intermediates and explains the origin of matching $\mathrm{T}=4$ icosahedral spike and NC lattices in released alphavirus virions (Cheng et al., 1995).

In the NC-centric model of alphavirus budding (Garoff et al., 2004), preformed icosahedral NLPs would provide a symmetric template for incorporation of spikes at the cell surface. In our study, 545 subvolumes of cytosolic NLPs without clear attachment to spikes at the PM were analyzed for any icosahedral symmetry that could direct virion assembly and budding (Fig. 3A, Fig. S5A). Following 3D-classification, respective sets of NLPs were refined with C5 symmetry, resulting in two NLP averaged structures (class I \& II), both lacking icosahedral symmetry (as seen in released virion NCs) or clear 5-fold symmetry of Cps (identified at the leading ends of budding intermediate NCs) (Fig. 3C, Fig. S4A). However, cytosolic NLP class II does display weak five-fold symmetry at one pole, raising the possibility that those NLPs were already interacting with spikes at the PM but their orientations in the tomograms prevented that observation. In addition, both cytosolic-NLP structures and the NC of the earliest

244 "docking" budding-intermediate are significantly smaller than spherical NCs of the lateststage budding intermediate ("tethered") and released virions (diameters $\sim 43 \mathrm{~nm}$ ) (Fig. S4A). The NC of the "docking" budding intermediate and cytosolic NLP exhibiting weak

247 five-fold symmetry at one end (class II) are both oblate spheroids, with long axis of $\sim 37$ $\mathrm{nm}$ and short axis between $31 \mathrm{~nm}$ and $33 \mathrm{~nm}$, respectively (Fig. 3B-C, Fig. S4A). 
250 The cytosolic NLP structure with no observable five-fold symmetry (class I) is roughly spherical with diameter $\sim 37 \mathrm{~nm}$ (Fig. 3C, S4A). For class I cytosolic NLPs, our result does not rule out that Cps can be arranged in assemblies with other non-icosahedral or non-five-fold symmetry. It is also possible that the low contrast of NCs in the cytosol results in inaccurate alignments and a non-symmetric average, though we consider this unlikely because of the following observations. To determine if weak five-fold symmetry in the cytosolic NLP class II average was located randomly on individual particles or the result of Cp:spike interactions, we mapped the refined subvolume particle orientations in 3D back to the originating tomograms. This confirmed that most particles within class II were positioned such that the five-fold organized-pole of the NLP was oriented towards the PM, presumably interacting with membrane-embedded spikes (Fig. S5). Weak fivefold symmetry within NCs prior to budding likely results from Cp:spike contacts at the PM, while the rest of the NLP structure lacks five-fold or icosahedral symmetry. Therefore, true cytosolic NLPs (class I) are structurally heterogeneous and lack icosahedral or local five-fold Cp organization. During budding, immature NLPs must undergo a significant structural maturation from an initial structurally heterogeneous RNA-Cp assembly to expanded, near-icosahedral viral NCs following ordering interactions with the icosahedral spike lattice.

\section{Assembly of spike lattices}

270 Structures of the budding-intermediates revealed a progressive spike-driven NC

271 morphogenesis, demonstrating the importance of the spike lattice in CHIKV particle

272 assembly. However, it was not known how the spike lattice acquires icosahedral

273 symmetry that reorganizes the NC. One possibility is that individual spikes bind to NCs

274 during budding and form lateral interactions with other spikes on the budding particle

275 surface (Forsell et al., 2000). Another proposal of alphavirus budding from preformed,

276 higher-order spike assemblies on the PM arose from previous reports of hexagonal

277 spike lattices as two-dimensional planes or 6-fold helical tubes (von Bonsdorff and 278 Harrison, 1978; Kononchik et al., 2009; Soonsawad et al., 2010). These alternative 279 spike assemblies were generated either by treating virions with detergent (von 
280 Bonsdorff and Harrison, 1978) or mutating both E1/E2 GPs (Kononchik et al., 2009), or 281 observed inside cytopathic vacuoles in virus-infected cells (Soonsawad et al., 2010).

282 Whether or not the alternative spike assemblies form at the PM of wt alphavirus-infected 283 cells, and their relevance to wt alphavirus assembly/budding, was unknown. In our 284 study, we visualized both spike organization near budding intermediates at the PM (Fig. 285 4), and structures of self-assembled spikes in wt CHIKV-infected cells in situ (Figs. 286 4\&5).

Spike organization at the PM prior to budding has never been observed in virus-infected cells due to the challenges in resolving relatively small macromolecules $(\sim 360 \mathrm{kDa})$ in different orientations in the unstained cell membrane with heterogeneous composition and high image background. In Volta phase plate cryoET data we were able to identify proteins near budding intermediates, potentially individual trimeric spikes based on shape and size, with no discernable high-order organization (dimer-, pentamer-, or hexamer-of-spikes) (Fig. 4A', A"). The lack of strict lateral organization between these proteins near the budding shells is significantly different from the spike organization identified in hexagonal spike lattices and the icosahedral-symmetric end of budding particles (Fig. 4B-C, Fig. S6).

Rare instances of near-planar sheets within thin extensions containing high density of spikes (Fig. 4B) were formed by hexagonal spike arrays without underlying NCs (Fig. 4B-B"). Side-views of spikes in the sheet lattices displayed characteristic spacing and conformation (Fig. 4B'). In addition, highly-curved tubular spike arrays were observed in multiple cellular contexts: at the base of budding particles (Fig. 5A), on extensions entirely devoid of NCs (Fig. 5C, Fig. S7), and on thin extensions loaded with budding particles (Fig. 4C, 5E). Subvolume averages of these tubes, achieved by applying helical rotations to compensate for the tomographic missing wedge, revealed helical organization of trimeric spikes arranged as hexagons (Fig. 5). No internal NC was observed inside any tube. Interestingly, the average diameter of the helical tubes varies 309 from 55 to $65 \mathrm{~nm}$, close to the diameter of the icosahedral CHIKV virion ( 70 nm) (Table 
bioRxiv preprint doi: https://doi org/10.1101/2021.10.14.464449; this version posted October 14,2021 . The copyright holder for this preprint (which was not certified by peer review) is the author/funder, who has granted bioRxiv a license to display the preprint in perpetuity. It is made available under aCC-BY-NC-ND 4.0 International license.

311 intermediate particles (Fig 4B", C"), while hexagons were observed on budding

312 particles, helical tubes (Fig. 4C', 5) and planar sheet lattices without interior NCs (Fig.

313 4B"). The correlation between underlying NC cores and pentagons of trimeric spikes in

314 the envelope lattice suggests that NC cores function to either directly promote five-fold

315 assembly of spikes and/or trigger membrane curvature formation for icosahedral

316 assembly.

317

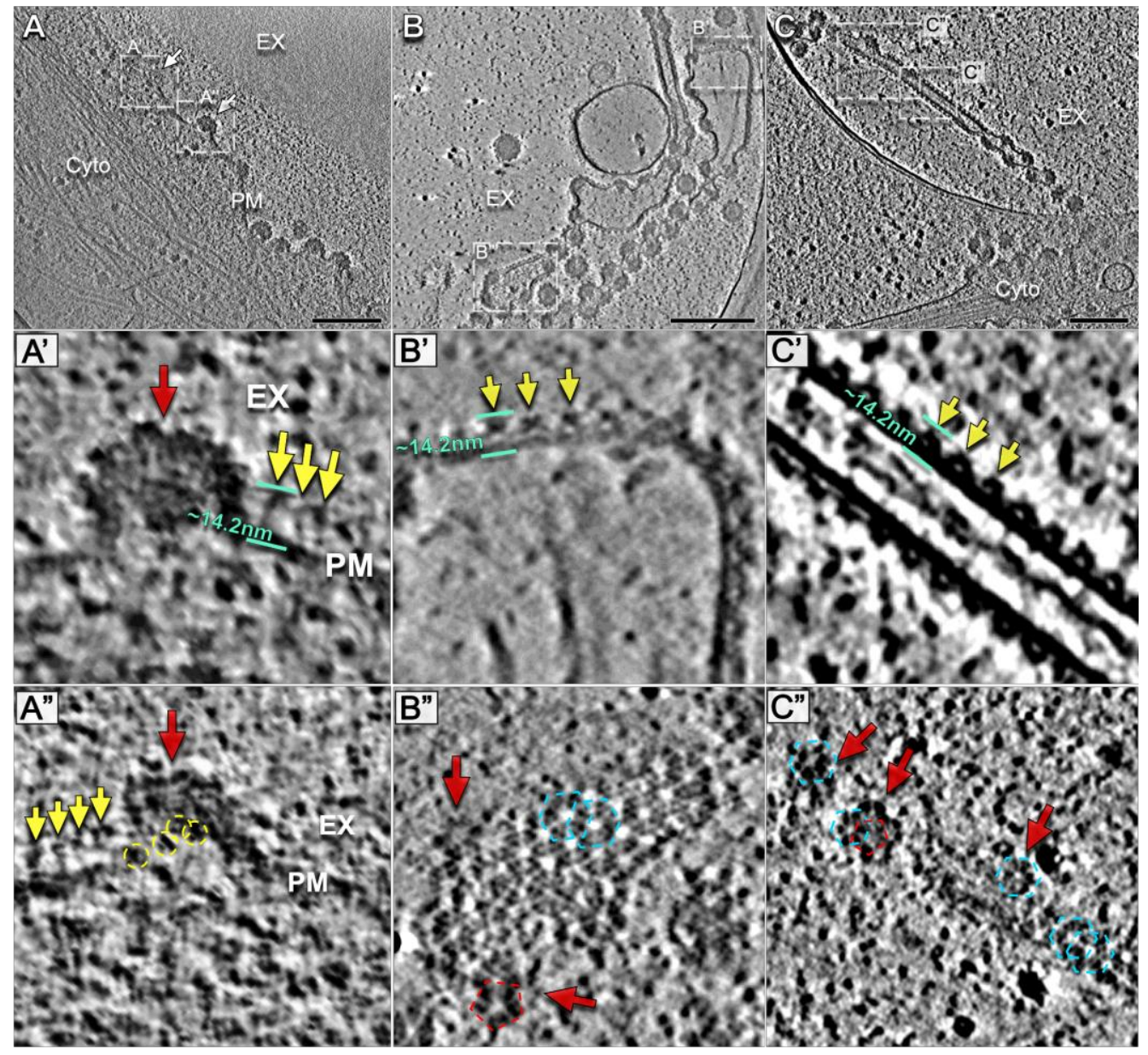

Figure 4. Spike organization on the virus-infected cell surface. Volta phase plate

tomogram slice images of $(A)$ cell periphery with budding intermediate particles (white arrows) and $(B, C)$ thin cell extensions enriched in linked, early-stage incomplete particles. Thin cell extensions include regions of planar sheets of spikes ( $B$, dashed white boxes) and tubular array of spikes ( $C$, dashed white box). (A') Detailed tomogram slice view displays apparent spikes

324 (yellow arrows) near a budding particle (red arrow). The distance between the crest of apparent 325 spikes and the inner leaflet of the PM (aquamarine lines) is measured to be $\sim 14.2 \mathrm{~nm}$. (A") Slice 
326 image displays apparent spikes as side-views (yellow arrows) and top-views (yellow circles)

327 near base of budding particle (red arrow). (see also Fig. S6) (B',C') Detailed tomogram slice

328 displays side views of PM with laterally organized spikes (yellow arrows) in planar sheets and

329 helical tubes, respectively. Distances between spike crest and inner leaflet of PM again

330 displayed in aquamarine. (B") Detailed tomogram slice with top view of a planar sheet reveals

331 spikes organized as a hexagonal lattice (blue dashed hexagons), with disruption in lattice near

332 budding virus particles (red arrows). Pentagon of spikes identified above NC (red dashed

333 pentagon). (C") Detailed tomogram slice of a helical spike tube-like structure reveals hexagonal

334 array of spikes (blue dashed hexagons), while both pentagons (red dashed pentagon) and

335 hexagons are observed on nearby linked, incomplete particles (red arrows).

337 Interestingly, compared with the spikes assembled into icosahedral or hexagonal

338 lattices, individual spikes at the base of budding particles not only lack ordered lateral

339 arrangement but also appear highly heterogeneous in shape and orientation (Fig. 4A-

340 A", Fig. S6). This suggests that spikes without lateral interactions and spikes assembled

341 into icosahedral or hexagonal lattices have different conformations. These results

342 explain why different epitope residues for specific mAbs were identified using cell-

343 surface displayed spikes, alphavirus-spike pseudotyped HIV-1 reporter viruses, and live

344 alphaviruses (Jin et al., 2015; Kim et al., 2021; Selvarajah et al., 2013). This could

345 suggest that spikes displayed on the surface of spike-transfected cells and

346 pseudoviruses have the same conformation as the individual spikes on the surface of

347 virus-infected cells. Future work leveraging advanced techniques to improve resolution

348 of heterogeneous biomolecules on noisy cellular background is warranted to resolve the 349 conformation(s) of individual spikes on the cell surface before virus assembly. 


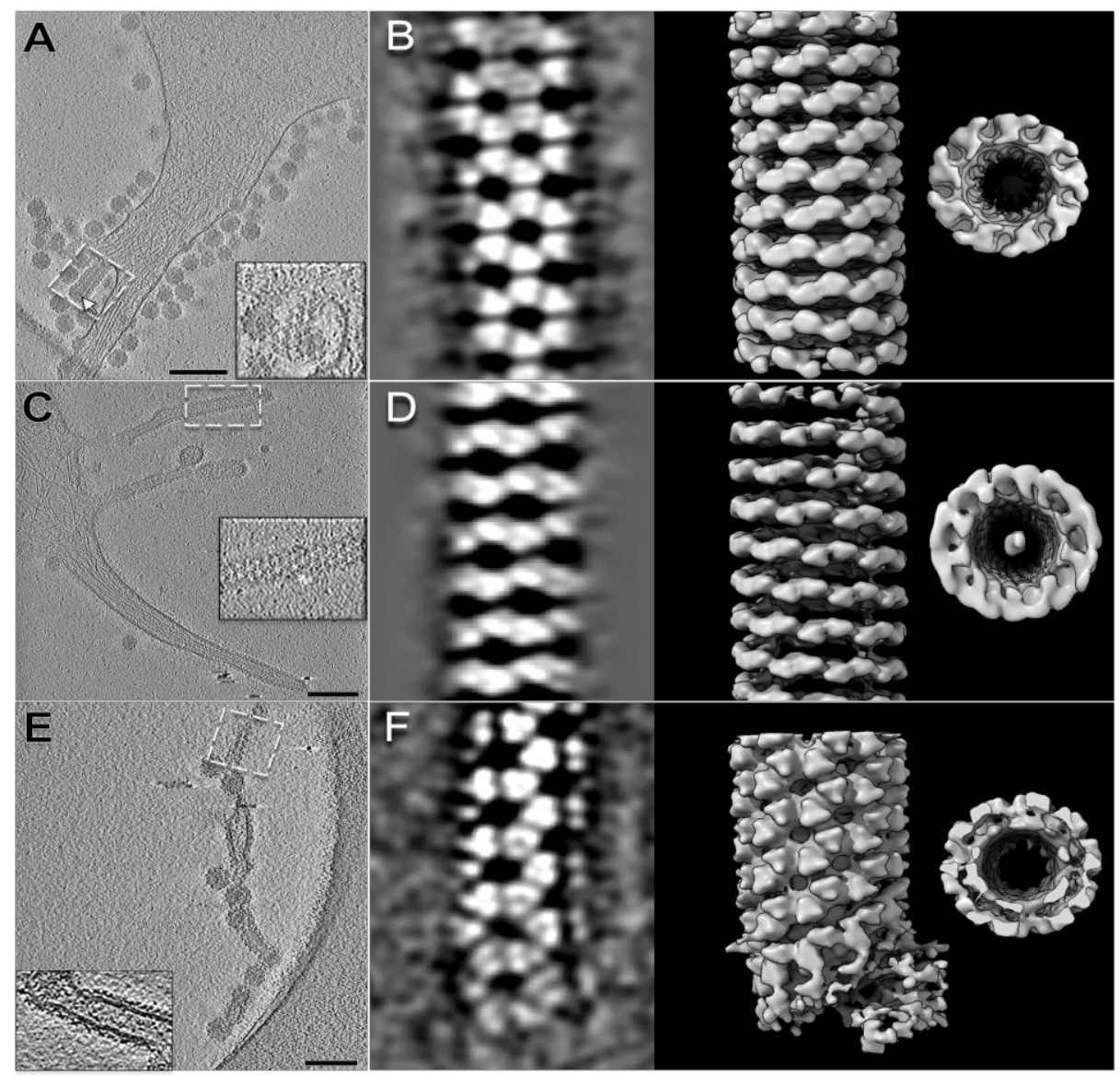

Figure 5. CHIKV envelope spikes arranged in hexagonal lattices form helical tubes in situ. $(A, C, E)$ Tomogram 2D slice images of cell extensions with membrane-embedded spike arrays (white dashed boxes) with enlarged inset views. Arrays form (A) at the base of a budding intermediate particle with NC at the leading end (white arrow), (C) on a cell extension without nearby NCs (see also Fig. S7), and as a (E) segment within a thin extension containing nearby budding viral particles. Scale bars $200 \mathrm{~nm}$. (B,D,F) 2D slice view of envelope lattice 3D subtomogram average (left) and density map (middle) (corresponding to A,C,E dashed white boxes) reveal helical arrays of trimeric spikes arranged as hexagon lattices. Density maps of each tube, rotated to view down the helical axis (right), revealed no underlying NCs below the spike lattice and membrane bilayer.

364 We previously reported that in addition to traditional NAb function in inhibiting virus entry, bivalent binding of NAbs to spikes at the outer surface of CHIKV-infected cells induces coalescence of spikes that inhibits virus assembly/budding (Jin et al., 2015, 
2018). To reveal if crosslinking NAbs induce budding arrest by disrupting self-assembly of spikes and/or icosahedral co-assembly of spikes and NC, we imaged CHIKV-infected cells treated with CHIKV-specific NAb C9 (Jin et al., 2015). Large numbers of cytosolic NLPs were observed docking to the inner leaflet of the PM without virus budding (Fig. 6 , movie S2), consistent with what we reported previously (Jin et al., 2018). Buddingarrested NLPs are characteristically flattened at the docking end below the near-planar

373 PM, where direct interactions are observed with cytosolic tails of C9-crosslinked spikes

374 (Fig. 6B, G). Side views of the PM revealed a layer of spike ectodomains above the lipid bilayer, and a second layer of dense protein density above the spikes, approximately $150-250 \AA$ from the inner leaflet of the PM (Fig. 6A-B). Proteins above the spike ectodomains, not seen in the regular CHIKV-infected cells, are presumably C9 IgGs bound to the previously reported epitope at the crest of spikes (Jin et al., 2015).

C9-bound trimeric spikes were readily detected in the tomograms, likely due to condensing of spikes crosslinked by $\mathrm{C} 9$, mass addition of bound IgG, and exclusion of other host membrane proteins (Fig. 6E-F, Movie S2). Spike-C9 complexes coalesced into large patches at micrometer scale (Jin et al., 2018). Neither ordered spike assemblies nor direct lateral interactions between spikes, like in spike hexagonal or icosahedral lattices, were observed, while densities between spikes with characteristic Y-shaped features of IgG molecules were readily detected (Fig. 6E-F). The clear boundaries of the coalescence of the spike-C9 complexes, with spikes often lined up, further suggests that spikes are cross-linked and spaced apart via bivalent binding of C9 IgGs. Additionally, subvolume averaging of 7,678 manually-picked, individual spikes yielded a low-resolution density map $\sim 24 \AA$ (0.143 FSC criterion) that approximately matches the atomic model of the CHIKV trimer (Fig. 6G). Based on the refined spike subvolume orientations, the median distance between nearest-neighbor spikes in the 393 C9-induced coalesced spike patches was determined to be $185.2 \AA$ (range from $98 \AA$ to $394309 \AA$ ) (Fig. 6H). This distance between centers of C9-linked spikes is mostly greater 395 than that between neighboring spikes in pentagons and hexagons on the surface of 396 mature icosahedral virions (117 $\AA$ and $120 \AA$ respectively) (Fig. 6I). This suggests C9 397 IgGs bridge between spikes and prevent formation of lateral interfaces required to form 
bioRxiv preprint doi: https://doi org/10.1101/2021.10.14.464449; this version posted October 14, 2021. The copyright holder for this preprint (which was not certified by peer review) is the author/funder, who has granted bioRxiv a license to display the preprint in perpetuity. It is made available under aCC-BY-NC-ND 4.0 International license.

398 pentagon and hexagon assemblies on the budding particle surface. The absence of 399 ordered spike assemblies in the spike-C9 coalescence further suggests that spikes are 400 unlikely to be delivered to the PM as pre-assembled lattices, arguing against what was 401 proposed from the observation of hexagonal spike lattice tubes in cytopathic vacuole 402 type-II in Semliki Forest virus-infected cells (Soonsawad et al., 2010).

403

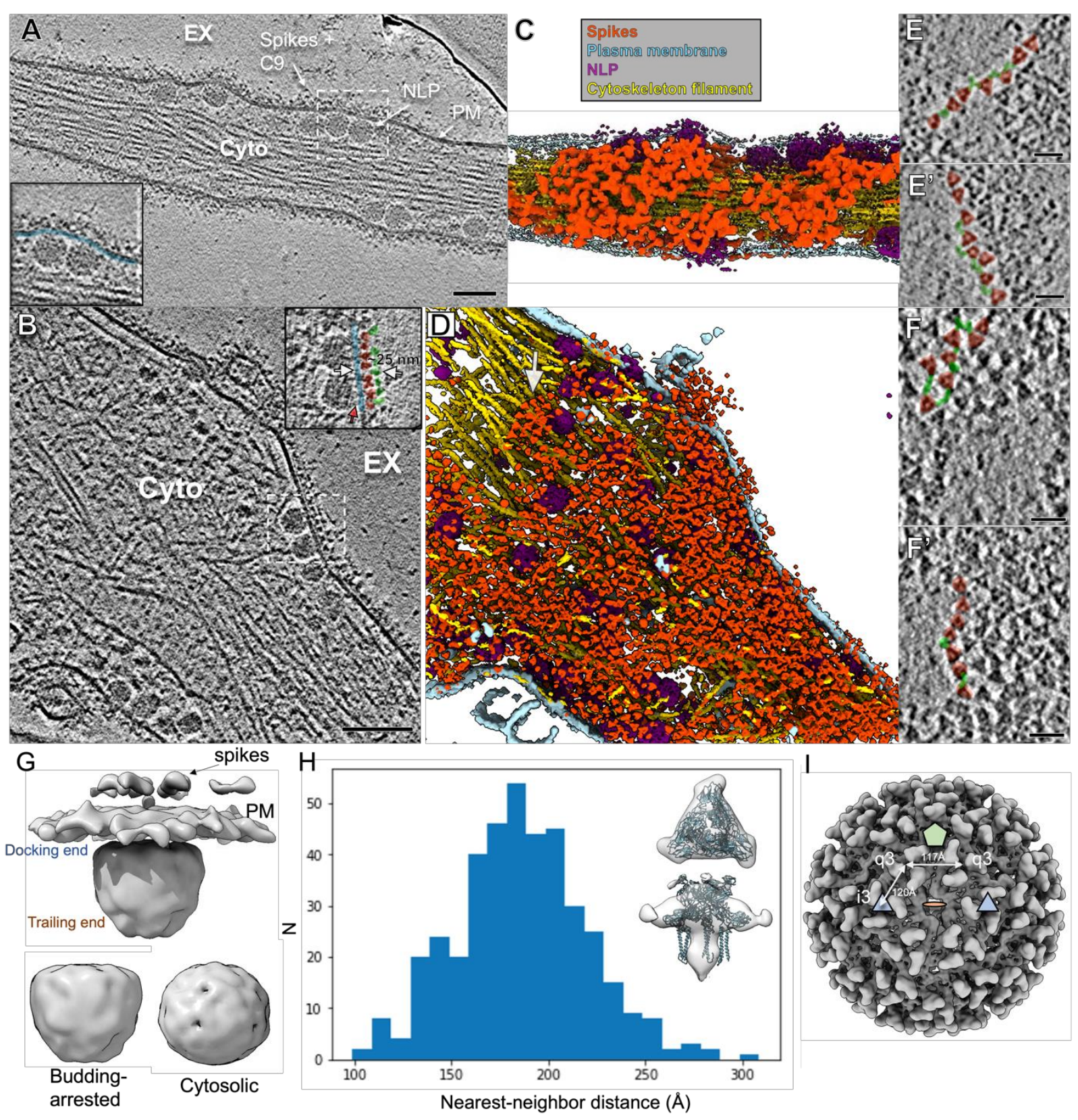


Figure 6. Neutralizing antibody C9 crosslinks spikes at the cell surface and induces coalescence of spike-C9 complexes.

(A-B) Individual slices of Volta phase plate cryoET tomograms displaying CHIKV-infected cells treated with NAb C9 revealed arrested NLPs at the PM inner leaflet and dense, coalesced spike-C9 complexes on the PM outer leaflet (inset images: docked NLPs interacting with spike intracellular tails (red arrow) at the PM inner leaflet (blue), with spike ectodomains (pink) bound

412 to NAb C9 (green) outside the cell. Scale bars 100nm. EX-extracellular, Cyto-cytosol. (C-D)

413 Corresponding 3D cellular annotations of tomograms (A-B), with trimeric spikes (orange-red),

414 PM (light blue), NLPs (purple) and cytoskeleton filaments (gold) colored. (E, E', F, F') Zoomed-

415 in top views of envelope spikes (orange-red) embedded in the PM with C9 (green) intercalating

416 trimeric spikes. Spikes with bridging C9 density (green) often arranged with clear, near-linear

417 boundaries. Scale bars $25 \mathrm{~nm}$. (G) Subvolume average of budding-arrested NLP below PM and

418 spikes. Docking end of egress-blocked NLPs is flattened in comparison to cytosolic NLP class I

419 (Fig. 3). (H) Plot of distance between C9-linked trimeric spike and nearest neighbor after

420 refinement of orientation for each extracted spike subvolume in representative tomogram shown

421 in (A). Low-resolution subvolume spike average shows general agreement with the CHIKV spike

422 atomic model (PDB:3J0C). (I) Distances on the virus particle between icosahedral-3-fold (i3)

423 spikes and quasi-3-fold spikes (q3), as well as q3-q3 spikes, displayed on the virus particle with

424 icosahedral 2-fold (orange disc), 3-fold (blue triangle) and 5-fold (green penton) for reference.

\section{Discussion}

428 Here we directly imaged CHIKV-infected cells to gain an enhanced understanding of

429 how Cp and spike proteins coordinate the assembly of icosahedral virus particles in

430 cellulo at the molecular level. As illustrated in Figure 7, immature, cytosolic NLPs

431 lacking observable symmetry promote nucleation of an icosahedral spike lattice at the

432 PM that transforms structurally heterogeneous, cytosolic NLPs into expanded,

433 icosahedral viral NC cores during virus budding. Lateral spike interactions progressively

434 form the icosahedral envelope lattice on the surface of budding particles, apparently

435 through sequential addition of individual spikes, rather than from rearrangement of

436 preformed spike arrays. Vertical spike:Cp interactions transmit symmetrical organization

437 from outer- to inner-shell. This outside-to-inside regulation is further supported by the

438 flattened docking end of budding-arrested NLPs apparently re-organized by NAb- 
crosslinked spikes on the PM surface (Fig. 6B, G). Our model supports both the existing paradigm that spike:Cp and spike:spike interactions are required for alphavirus assembly and a model of alphavirus budding where spike shell assembly provides the driving force for icosahedral particle formation (Fig. 7).

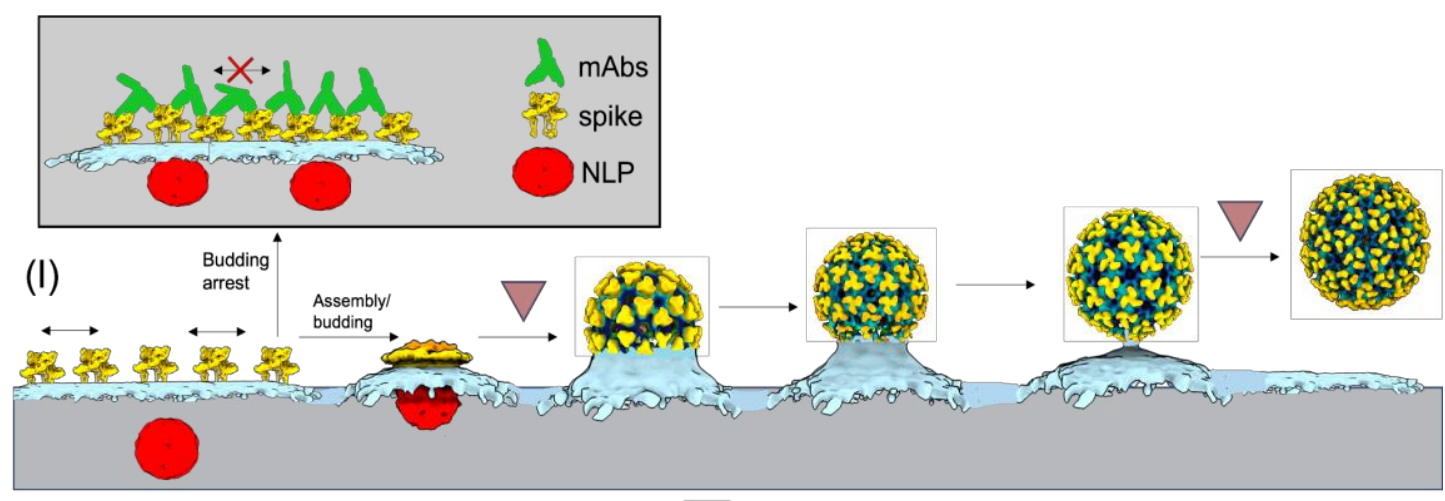

\section{Figure 7. Mechanistic model of alphavirus budding and assembly.}

At the CHIKV-infected cell surface, immature non-icosahedral NLPs and membrane-embedded spikes converge. Subsequent virus budding $(I)$ is predicated on assembly of the icosahedral spike lattice that enwraps NLPs and reorganizes them into icosahedral NCs through sequential spike:Cp interactions. Rate-limiting steps to particle formation likely occur at early- and latestages associated with assembly of the first half of the virions and membrane scission following completion of full virions, respectively (upside-down red triangles). Released virions contain near-icosahedral spike and NC layers, with local disruptions in the lattices likely related to membrane scission and virus release from the PM. Binding of mAbs to exposed spike surfaces at the PM (boxed) inhibits virus biogenesis by preventing formation of the curved, icosahedral spike shell. (II) Spikes can self-assemble into non-icosahedral structures, giving rise to alternative assembly products, including $(\mathrm{a}, \mathrm{c})$ helical tubes formed by spike hexagons, (b) thin extensions of linked, incomplete particles and (d) planar hexagonal sheets of spikes. 
459 In the CHIKV-infected cell system, we were able to capture snapshots of virus budding 460 from the PM, showing many distinct assembly states. While cryoEM is now commonly 461 used to produce 3D reconstructions, the single-molecule nature of imaging also allows

462 for analysis of a macromolecule's entire conformational landscape at atomic detail 463 through static images of its many individual states. Analysis of CHIKV assembly states 464 through the entire progression of virion budding differs significantly from past in situ 465 cryoET and subvolume averaging studies of non-enveloped viruses with apparently 466 distinct assembly intermediate populations in the cells (Dai et al., 2013; Sutton et al., 467 2020; Vijayakrishnan et al., 2020). Our discrete-state method of classifying and 468 averaging those individual budding states with compositional and conformational 469 heterogeneity into ensemble 3D classes is an important step in studying progression of 470 a transient assembly process on the cell membrane. In addition to capturing assembly 471 intermediates which are not amenable to in vitro purification, our study shows the 472 usefulness of using cryoET to directly derive models of dynamic biological processes in 473 the cell. From the population of particles in each of the 12 budding classes (Fig. 2), it 474 was possible to derive a model of non-uniform budding progression. This model 475 suggests that formation of the first half of the budding shell around the NC, and final 476 scission of the budding membrane neck to release a fully-assembled virion, are likely 477 energetically unfavorable processes and rate-limiting steps in the budding process in 478 situ.

480 In analyzing the contribution of spikes and NCs to assembly of icosahedral particles, our 481 study suggests cytosolic NLPs are roughly spherical but lack pre-formed symmetric 482 organization and therefore do not directly guide icosahedral placement of spikes at the 483 PM during virus budding. Instead, NLPs promote efficient budding of infectious particles 484 by serving as a rough scaffold with suitable curvature to guide assembly of the 485 icosahedral spike lattice. This function in controlling particle size and architecture is 486 reminiscent of dsDNA bacteriophage scaffolding proteins that control the 487 hexamer:pentamer ratio during icosahedral capsid assembly (Chen et al., 2011). Our 488 discovery conflicts with the $\mathrm{Cp}$-centric alphavirus assembly/budding model derived from 489 studies with biochemically reconstructed CLPs (Cheng and Mukhopadhyay, 2011; 
490 Ferreira et al., 2003; Mukhopadhyay et al., 2002; Snyder et al., 2011; Wang et al., 491 2015). Future studies examining early assembly of Cp-gRNAs into cytosolic NLPs and 492 intracellular NLP trafficking using focused ion beam milling (Rigort et al., 2012) of 493 infected cells can provide additional insights into NC morphogenesis. Interestingly, 494 asymmetric NCs serving as a scaffold for spike-driven virus budding can be a common 495 mechanism of particle formation among flaviviruses and alphaviruses (Ferlenghi et al., 496 2001; Tan et al., 2020).

Our study for the first time demonstrated that self-assembly of spikes produced rare, alternative hexagonal lattices on the PM in situ (Fig. 4B-C), in addition to driving budding of the predominant icosahedral virions (Fig. 4A). The hexagonal spike lattice coated vesicles shed off from virus-infected cells (Fig. S7D), may function like the capsidless subviral particles formed by transmembrane glycoproteins of other virus families (Allison et al., 2003; Bruss and Ganem, 1991; Ferlenghi et al., 2001; de Haan et al., 1998; Heilingloh and Krawczyk, 2017; Stange et al., 2008; Wang et al., 2009). In an infected host, subviral particles may help infectious virus particles escape from host immune responses by absorbing specific antiviral antibodies as virus decoys (Heilingloh and Krawczyk, 2017; Vaillant, 2021). The long extensions from the cell periphery, in form of helical spike tubes or thin strings of incomplete particles might promote virus cell-to-cell transmission like reported for retroviruses (Nikolic et al., 2011; Sherer and Mothes, 2008; Sowinski et al., 2008), although using different mechanisms. Infection from the incomplete particle strings, or often-observed released multi-core particles,

512 would result in high local multiplicity of infection (MOI) that usually promotes virus 513 infection by saturating host restriction factors (Bieniasz, 2004; Yan and Chen, 2012), 514 and contributes to modulation of viral pathogenesis by maintaining genetic diversity 515 (Sanjuán, 2021; Vignuzzi and López, 2019). Future functional studies of alternative 516 assemblies of alphaviruses, both in vitro and in vivo, are warranted.

518 We previously reported that anti-alphavirus NAbs are able to inhibit virus release in 519 addition to their classical function in neutralizing virus entry, and this anti-release function depends on bivalent binding of NAb lgGs to viral spikes (Fox et al., 2015; Jin et 
521 al., 2015; Williamson et al., 2021). Spikes crosslinked by NAbs coalesce into membrane 522 patches that prevent membrane envelopment around attached NLPs, therefore blocking 523 virus budding (Jin et al., 2018). Based on these previous studies we proposed that 524 alphavirus assembly/budding is a potential new target for antiviral therapies. Detailed 525 molecular mechanisms of spatially- and temporally-regulated assembly of alphavirus 526 particles in the cellular environment, as well as the mechanism of NAb-induced 527 alphavirus budding inhibition, will facilitate the design of novel anti-budding therapies. In 528 the current study, we for the first time characterized in-situ orchestrated assembly of 529 two-layer icosahedral particles driven by lateral spike interactions and demonstrated 530 that intercalation of NAbs between spikes prevented lateral spike-spike interactions 531 required to assemble hexagonal or icosahedral lattices (Fig. 6). Although we discovered 532 the anti-budding functions for anti-CHIKV antibodies using NAbs binding to E2 (Fox et 533 al., 2015; Jin et al., 2015), the detailed mechanism revealed in this study suggests that 534 any intercalating molecule that locks spikes in a conformation preventing lateral spikespike interactions could inhibit alphavirus budding. Targeting conserved regions exposed on individual spikes with antibody or other cross-linking molecules can serve

537 as pan-alphavirus antivirals without the need to neutralize virus entry. Two recent 538 studies published while this work is in preparation support our hypothesis (Kim et al., 539 2021; Williamson et al., 2021), where cross-reactive non-neutralizing mAbs targeting 540 the conserved region in alphavirus E1 are able to inhibit virus budding from arthritogenic 541 to encephalitogenic alphaviruses and provide in vivo pan-protection against alphavirus 542 infection.

544 It is also conceivable that blocking the spike lateral interfaces that are exposed on the 545 individual spike surface, but concealed in spike lattices, can also prevent icosahedral 546 spike lattice formation and subsequent virus budding. In addition to IgG-like large 547 biomolecules, small molecules designed to accurately target spike-spike interfaces can 548 potentially be developed as anti-alphavirus therapies. In the current study we 549 demonstrated that spike-Cp vertical interactions initiate the icosahedral virus 550 assembly/budding. Interestingly, microinjection of synthesized peptide corresponding to 551 the E2 cytoplasmic domain that interacts with NC was reported to successfully inhibit 
552 SINV and SFV budding (Kail et al., 1991). It is conceivable that membrane penetrating

553 molecules that interfere with spike-Cp interactions could functionally inhibit alphavirus

554 biogenesis and spreading. Targeting both the extracellular domain and intracellular tail

555 of spikes concurrently will reduce the chance of escaping for alphaviruses that have

556 high mutation rate and often escape from antivirals quickly. Taken together, our current

557 study provides valuable structural information on developing intervening molecules that

558 block alphavirus assembly/budding in two ways: preventing lateral interactions between

559 spikes from outside and/or uncoupling NC and spike assembly from inside of virus-

560 infected cells.

Methods

564 KEY RESOURCES TABLE

\begin{tabular}{|c|c|c|}
\hline REAGENT or RESOURCE & SOURCE & IDENTIFIER \\
\hline \multicolumn{3}{|l|}{ Antibodies } \\
\hline C9 & $\begin{array}{l}\text { (Selvarajah et al., } \\
\text { 2013) }\end{array}$ & $\mathrm{N} / \mathrm{A}$ \\
\hline \multicolumn{3}{|l|}{ Bacterial and virus strains } \\
\hline CHIKV vaccine strain $181 /$ clone 25 & BEl Resources & Cat. \#NR-13222 \\
\hline \multicolumn{3}{|c|}{ Chemicals, peptides, and recombinant proteins } \\
\hline Fibronectin solution & Sigma-Aldrich & Cat. \#F1141 \\
\hline \multicolumn{3}{|l|}{ Deposited data } \\
\hline Budding intermediate maps & This manuscript & $\begin{array}{l}\text { EMDB-25094, -25095, -25096, } \\
-25097\end{array}$ \\
\hline Cytosolic NLP maps & This manuscript & EMDB-25098, -25099 \\
\hline Released virion map & This manuscript & EMDB-25093 \\
\hline \multicolumn{3}{|l|}{ Experimental models: Cell lines } \\
\hline Human U2OS cell line & ATCC & Cat. \#HTB-96 \\
\hline BHK21 cell line & ATCC & cat. \#CCL-10 \\
\hline \multicolumn{3}{|l|}{ Software and algorithms } \\
\hline Serial EM & $\begin{array}{l}\text { (Mastronarde, } \\
2003,2005)\end{array}$ & $\begin{array}{l}\text { http://bio3d.colorado.edu/Seria } \\
\text { IEM/ }\end{array}$ \\
\hline Tomo4 & $\begin{array}{l}\text { ThermoFisher } \\
\text { Scientific }\end{array}$ & $\begin{array}{l}\text { https://www.thermofisher.com/ } \\
\text { us/en/home/electron- } \\
\text { microscopy/products/software- } \\
\text { em-3d-vis/tomography- } \\
\text { software.html }\end{array}$ \\
\hline
\end{tabular}




\begin{tabular}{|l|l|l|}
\hline IMOD & $\begin{array}{l}\text { (Kremer et al., } \\
1996)\end{array}$ & $\begin{array}{l}\text { https://bio3d.colorado.edu/imo } \\
\mathrm{d} /\end{array}$ \\
\hline MotionCor2 & $\begin{array}{l}\text { (Zheng et al., 2017) } \\
\text { https://emcore.ucsf.edu/ucsf- } \\
\text { software }\end{array}$ \\
\hline EMAN2 & (Chen et al., 2019) & $\begin{array}{l}\text { https://blake.bcm.edu/emanwi } \\
\text { ki/EMAN2 }\end{array}$ \\
\hline UCSF Chimera & $\begin{array}{l}\text { (Pettersen et al., } \\
2004)\end{array}$ & $\begin{array}{l}\text { https://www.cgl.ucsf.edu/chim } \\
\text { era/ }\end{array}$ \\
\hline UCSF ChimeraX & $\begin{array}{l}\text { (Pettersen et al., } \\
2021)\end{array}$ & $\begin{array}{l}\text { https://www.rbvi.ucsf.edu/chim } \\
\text { erax/ }\end{array}$ \\
\hline
\end{tabular}

\section{Lead Contact}

568 Further information and requests for resources and reagents should be directed to and 569 will be fulfilled by the lead contact, Wah Chiu (wahc@stanford.edu), Jing Jin 570 (jjin@vitalant.org).

\section{Data and code availability}

573 Cryo-EM maps reported in this study have been deposited in the Electron Microscopy 574 Data Bank (EMDB) under the following accession codes: EMDB-25093 (released virion), 575 EMDB-25094, -25095, -25096, -25097 (budding intermediates), and EMDB-25098, 57625099 (cytosolic NLPs).

578 EXPERIMENTAL MODEL AND SUBJECT DETAILS

\section{Cell cultures}

581 Human bone epithelial cell line U2OS cells (Cat. \#HTB-96) is a female cell line purchased 582 from American Type Culture Collection (ATCC). Hamster fibroblast cell line BHK21 cells 583 (Cat. \#CCL-10) were purchased from ATCC. Cells were maintained at $37^{\circ} \mathrm{C}$ with $5 \%$ 584 humidified $\mathrm{CO}_{2}$ in DMEM (Invitrogen) supplemented with penicillin and streptomycin, 10 
585 mM HEPES, non-essential amino acids, and 10\% FBS (Hyclone). CHIKV vaccine strain 586 181/clone 25 (CHIKV-181) was amplified in BHK21 cells.

Virus Strain

589 CHIKV vaccine strain 181/clone 25 (CHIKV-181) was amplified in BHK21 cells.

\section{METHODS DETAILS}

\section{Cell infection and vitrification}

593 U2OS cells grown on fibronectin-coated gold 200 mesh R2/2 grids (Quantifoil) were 594 infected with $\mathrm{CHIKV}-181$ at an $\mathrm{MOI}$ of 50 for an incubation period of $8 \mathrm{hrs}$. In the case of 595 neutralizing antibody-treated cells, after $3 \mathrm{hrs}$ of infection, the grids were washed 596 extensively and incubated with $5 \mathrm{ug} / \mathrm{mL}$ NAb C9 for an additional $5 \mathrm{hrs}$. The grids were 597 then washed with PBS and a solution of $10 \mathrm{~nm}$ BSA gold tracer (Cat. \#25486, EMS)

598 was added directly prior to vitrification. Grids were blotted and plunged into liquid ethane 599 using the LEICA EMGP plunge freezer device. Grids were stored under liquid nitrogen conditions until required for data collection.

\section{Acquisition and processing of cryo-ET tilt series}

603 Grids of vitrified virus-infected cells were imaged on two instruments: (1) a Titan Krios 604 microscope (ThermoFisher) operated at 300kV with post-column energy filter (20eV) 605 and K2 Summit detector (Gatan) with a calibrated pixel size of $2.72 \AA$ and (2) a Talos

606 Arctica (ThermoFisher) operated at 200kV with post-column energy filter (20eV) andK2 607 Summit detector with calibrated pixel size of $3.54 \AA$. Single-axis, bi-directional tilt series 608 were collected using SerialEM software with low-dose settings and defocus range of -3 609 to $-5.5 \mu \mathrm{m}$. For data of CHIKV-181-infected cells collected with the Titan Krios, a total 610 cumulative dose of $110 \mathrm{e}^{-/ \mathrm{A}^{2}}$ was applied to the specimen, while for data collected with 611 Talos Arctica, the total average dose at the specimen was $90 \mathrm{e}^{-} / \mathrm{A}^{2}$. In both cases the 612 electron dose was distributed over 51 tilt images, covering an angular range of $-50^{\circ}$ to $613+50^{\circ}$, with an angular increment of $2^{\circ}$. Additional data collection on both electron 614 microscopes was collected using a Volta phase plate, whereby the objective aperture 
615 was removed, phase plate inserted and activated, and tilt series collected under the

616 above conditions. The activated Volta phase plate was operated at phase shift 0.3-0.6

617 radians as measured by AutoCTF software (ThermoFisher). The motion between

618 frames of each tilt image in the tilt series was corrected using MotionCor2 software

619 (Zheng et al., 2017). Tilt images were compiled, automatically aligned and

620 reconstructed using EMAN2 software (Chen et al., 2019). In total, 144 tomograms were

621 judged as sufficient for further analysis from the Titan Krios data collections and 20

622 tomograms from the Talos Arctica data collections. A summary of the Cryo-ET data

623 collection can be found in Supplementary Table 1.

625 For analysis of CHIKV-181-infected cells treated with NAb C9, 61 single-axis, bi626 directional tilt series were collected on the Titan Krios microscope operated at 300kV

627 with post-column energy filter and K2 Summit detector and calibrated pixel size of 628 2.72A. Data was acquired using SerialEM software with low-dose settings and defocus 629 range of -3 to $-5.5 \mu \mathrm{m}$. Tilt series were collected with a total cumulative electron dose of

$630120 \mathrm{e}^{-} / \mathrm{A}^{2}$ distributed over 51 tilt images, again covering an angular range of $-50^{\circ}$ to $+50^{\circ}$, 631 with an angular increment of $2^{\circ}$. Data was exclusively collected using an activated Volta 632 phase plate, with phase shift targeted in the range 0.3-0.6 $\pi$ radians. 51 tomograms

633 were judged as sufficient for further analysis, based on achieved phase shift and

634 tomogram reconstruction quality, and were used for subvolume analysis.

Classification of budding intermediate subvolumes

637 Subvolume analysis steps were performed using the EMAN2 Tomo pipeline (Chen et 638 al., 2019). CTF estimation for each tilt image was performed using the EMAN2 program 639 e2spt_tomoctf.py. 1,918 budding intermediate particles were manually picked using the 640 EMAN2 3D slice picker and extracted into subvolumes with $x 4, x 2, x 1$ binning. 50 high641 SNR particles ( $\mathrm{x} 4$ binning) were picked from the dataset for each of three rough stages 642 of budding (early-, mid-, and late-) for initial model generation. The initial model for each 643 budding class was produced using the EMAN2 initial model generation program 644 e2spt_sgd.py, first imposing c1 symmetry and running 5 iterations. After aligning the C1 645 initial models to the symmetry axes, 5 additional iterations were run with C5 symmetry 
646 imposed for each. These three maps were then used as initial models for subtomogram

647 multi-reference refinement (e2spt_refinemulti.py).

649 The full dataset of 1,918 budding-intermediate subvolumes (x4 binning) was input into 650 EMAN2 multi-reference refinement with 10 initial models (three copies of early-, three 651 copies of mid-, and four copies of late-budding models described above) and run for 12 652 iterations, imposing C5 symmetry and limiting resolution to $40 \AA$ for alignments. Due to 653 poor convergence of the earliest-budding classes, all budding particles in the 654 tomograms were re-picked with two points defining an initial budding orientation: one at 655 the center of NC and one at the apex of the budding shell. Multi-reference refinement of 656 the pre-oriented subvolumes was repeated as described above, with a refinement 657 angular difference constraint of $30^{\circ}$ to prevent particle "flipping" from the initial and 658 rough budding orientation. If a resulting class displayed budding virus structural features 659 with sufficient particle count, those particles were subjected to further classification with 660 either two or three low-passed versions of the class average as initial references. In this way, particles within five of the $103 \mathrm{D}$ classes were subjected to a second round of

662 multi-reference refinement for further identification of budding conformations, with 663 refinement parameters described above (Fig. S3). Between the two rounds of 664 classification, 12 different 3D budding structures were determined in total. Subvolume 665 particles within "junk" class averages lacking interpretable structure were viewed in the 666 original 3D tomograms, revealing these particles covered a wide range of budding 667 levels and were typically located near high density gold fiducials that biased the 668 alignment.

\section{Subtomogram averaging of budding intermediates, released virions and NLPs}

671 For each of the five budding intermediate 3D classes displaying low-resolution 672 icosahedral features, particles were re-extracted (x4, x2, x1 binning) for subtomogram 673 refinement (e2spt_refine.py). For each class, 4-6 iterations of refinement were 674 performed for each binned (x4, x2, x1) particle set, imposing C5 symmetry at each step 675 and following gold-standard protocol: all particles were split into two independent 676 subsets and resolution measured by Fourier shell correlation ( 0.143 FSC criterion) of 
677 the two density maps. Following subtomogram refinement of the least-binned particle

678 set for each class, 2 iterations of sub-tilt refinement (e2spt_tiltrefine.py) with imposed

679 C5 symmetry were performed to produce final budding-intermediate subvolume

680 averages. A summary of the CryoET data collection and subtomogram analysis of viral 681 intermediates can be found in Table S1.

683 Subtomogram averaging of released virions was performed by manually picking and 684 extracting 521 released particles (x4, x2, x1 binning) into subvolumes, followed by 685 EMAN2 3D refinement and sub-tilt refinement. An initial reference for 3D refinement 686 was generated from 50 high SNR particles with different defocuses using EMAN2, with 687 C5 symmetry imposed as described previously. 3D refinement was performed with C5 symmetry imposed, working from $\times 4$ to $x 2$ to $x 1$ binned subvolumes as resolution improved. After visual observation of icosahedral structure in the map, icos. symmetry was applied during final sub-tilt refinement of $x 1$ binned subvolumes. This resulted in a converged map with pixel size $2.72 \AA$ /pixel and resolution (0.143 FSC criterion) of $8.2 \AA$.

For subtomogram averaging of NLPs, 545 NLPs apparently within the cytosol of virusinfected cell tomograms were manually picked using the EMAN2 3D slice picker and extracted (x4binning) into subvolumes. 50 high SNR particles with varying defocus were used to generate an initial reference with C5 symmetry as described above. Multireference refinement of the 545 NLPs (x4 binning) was performed with three classes and similar refinement parameters described above for budding intermediate classification, without the angular difference constraints. This resulted in two cytosolic NLP 3D classes (class I \& II) with interpretable structure (Fig. S3). Additional 3D refinements of particles within those two respective classes, with imposed C5

702 symmetry, resulted in maps with resolutions of $47.6 \AA$ (class I) and $43.5 \AA$ (class II)

703 (Gold-standard, 0.143 FSC criterion). The refined orientations of cytosolic NLPs within 704 one class displaying local five-fold symmetry (class II, Fig. 3, S4) were mapped back in

$7053 \mathrm{D}$ to originating tomogram reconstructions using EMAN2 program

706 e2spt_mapptclstotomo.py. 


\section{Subvolume analysis of NAb-crosslinked spikes and budding-arrested NLPs}

709 For analysis of the C9-treated CHIKV-181-infected cells, 7,678 individual spikes were

710 automatically picked from tomograms based on a low-resolution reference and judged

711 individually for false positives. Any additional spikes in the tomogram were picked

712 manually. This extensive manual picking protocol was meant to ensure all spikes were

713 properly extracted for nearest-neighbor distance analysis. 3D subvolumes (x4, x2

714 binning) of each spike were then extracted and a c3-symmetric initial model was built

715 from a subset of 500 (x4 binning) high SNR particles using the reference-free initial

716 model program in EMAN2 (e2spt_sgd.py). The full set of 7,678 (x4, x2 binned) spike

717 particles was then subjected to iterative 3D subtomogram refinement (e2spt_refine.py)

718 with C3 symmetry imposed until no improvement in refined orientations was achieved.

719 The final converged average map had resolution 24.4A (Gold-standard, 0.143 FSC

720 criterion) and pixel size 5.44A/pixel. The Euclidean distance between each refined spike

721 and its nearest neighbor in the dataset was determined using the refined center-of-mass

722 orientations of spike subvolumes in each tomogram.

723

724 From the same tomograms, 1,727 budding-arrested NLPs were manually picked,

725 extracted into subvolumes (x4 binned) and an initial model was generated from 50

726 particles in the dataset as described above with C5 symmetry imposed. 3D refinement

727 of the 1,727 NLP subvolumes, imposing C5 symmetry, resulted in a converged map

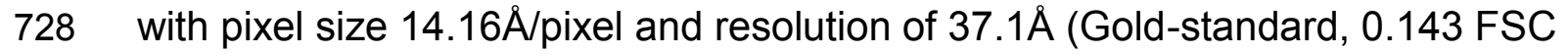

729 criterion).

730

731 Visualization, figure generation and model docking were performed in UCSF ChimeraX

732 and its built-in tools (Pettersen et al., 2021).

734 Acknowledgements

735 We thank SLAC National Accelerator Laboratory for access and support of these

736 studies, and all SLAC cryoEM staff for technical support and assistance. We also thank

737 Dr. Muyuan Chen for helpful discussions and providing technical advice in data 
738 analysis. This research was supported by the NIH grants R01Al148382, P01Al120943

739 and S10OD021600 (to W.C.) and R01Al119056 (to G.S.).

\section{Conflicts of Interest}

742 All authors declare no competing interest.

743

\section{Author Contributions}

745 D.C., J.J., and W.C. designed the study. D.C and J.J. performed cryoEM sample

746 preparation and collected cryoET data. D.C. performed 3D reconstruction and

747 subtomogram averaging. D.C, J.J., M.S., and W.C. analyzed the data. D.C., J.J., and

748 W.C. wrote the manuscript with support from all co-authors. 


\section{REFERENCES}

751 Allison, S.L., Tao, Y.J., O’Riordain, G., Mandl, C.W., Harrison, S.C., and Heinz, F.X.

752 (2003). Two distinct size classes of immature and mature subviral particles from tick753 borne encephalitis virus. J. Virol. 77, 11357-11366.

754 Basore, K., Kim, A.S., Nelson, C.A., Zhang, R., Smith, B.K., Uranga, C., Vang, L., 755 Cheng, M., Gross, M.L., Smith, J., et al. (2019). Cryo-EM Structure of Chikungunya

756 Virus in Complex with the Mxra8 Receptor. Cell 177, 1725-1737.e16.

757 Bieniasz, P.D. (2004). Intrinsic immunity: a front-line defense against viral attack. Nat. 758 Immunol. 5, 1109-1115.

759 Birdwell, C.R., Strauss, E.G., and Strauss, J.H. (1973). Replication of Sindbis virus. III. 760 An electron microscopic study of virus maturation using the surface replica technique. 761 Virology 56, 429-438.

762 von Bonsdorff, C.H., and Harrison, S.C. (1978). Hexagonal glycoprotein arrays from 763 Sindbis virus membranes. J. Virol. 28, 578-583.

764 Brown, R.S., Anastasakis, D.G., Hafner, M., and Kielian, M. (2020). Multiple capsid 765 protein binding sites mediate selective packaging of the alphavirus genomic RNA. Nat. 766 Commun. 11, 4693.

767 Bruss, V., and Ganem, D. (1991). The role of envelope proteins in hepatitis B virus 768 assembly. Proc. Natl. Acad. Sci. U. S. A. 88, 1059-1063.

769 Chen, D.-H., Baker, M.L., Hryc, C.F., DiMaio, F., Jakana, J., Wu, W., Dougherty, M., 770 Haase-Pettingell, C., Schmid, M.F., Jiang, W., et al. (2011). Structural basis for 771 scaffolding-mediated assembly and maturation of a dsDNA virus. Proc. Natl. Acad. Sci. 772 U. S. A. 108, 1355-1360.

773 Chen, L., Wang, M., Zhu, D., Sun, Z., Ma, J., Wang, J., Kong, L., Wang, S., Liu, Z., Wei, 774 L., et al. (2018). Implication for alphavirus host-cell entry and assembly indicated by a $7753.5 \AA$ resolution cryo-EM structure. Nature Communications 9. 
776 Chen, M., Bell, J.M., Shi, X., Sun, S.Y., Wang, Z., and Ludtke, S.J. (2019). A complete

777 data processing workflow for cryo-ET and subtomogram averaging. Nat. Methods 16,

778 1161-1168.

779 Cheng, F., and Mukhopadhyay, S. (2011). Generating enveloped virus-like particles 780 with in vitro assembled cores. Virology 413, 153-160.

781 Cheng, R.H., Kuhn, R.J., Olson, N.H., Rossmann, M.G., Choi, H.K., Smith, T.J., and 782 Baker, T.S. (1995). Nucleocapsid and glycoprotein organization in an enveloped virus. 783 Cell 80, 621-630.

784 Choi, H.K., Tong, L., Minor, W., Dumas, P., Boege, U., Rossmann, M.G., and Wengler, 785 G. (1991). Structure of Sindbis virus core protein reveals a chymotrypsin-like serine 786 proteinase and the organization of the virion. Nature 354, 37-43.

787 Choi, H.K., Lu, G., Lee, S., Wengler, G., and Rossmann, M.G. (1997). Structure of 788 Semliki Forest virus core protein. Proteins 27, 345-359.

789 Dai, W., Fu, C., Raytcheva, D., Flanagan, J., Khant, H.A., Liu, X., Rochat, R.H., Haase790 Pettingell, C., Piret, J., Ludtke, S.J., et al. (2013). Visualizing virus assembly 791 intermediates inside marine cyanobacteria. Nature 502, 707-710.

792 Danev, R., Buijsse, B., Khoshouei, M., Plitzko, J.M., and Baumeister, W. (2014). Volta 793 potential phase plate for in-focus phase contrast transmission electron microscopy.

794 Proc. Natl. Acad. Sci. U. S. A. 111, 15635-15640.

795 Ferlenghi, I., Clarke, M., Ruttan, T., Allison, S.L., Schalich, J., Heinz, F.X., Harrison, 796 S.C., Rey, F.A., and Fuller, S.D. (2001). Molecular organization of a recombinant 797 subviral particle from tick-borne encephalitis virus. Mol. Cell 7, 593-602.

798 Ferreira, D., Hernandez, R., Horton, M., and Brown, D.T. (2003). Morphological variants 799 of Sindbis virus produced by a mutation in the capsid protein. Virology 307, 54-66.

800 Forsell, K., Griffiths, G., and Garoff, H. (1996). Preformed cytoplasmic nucleocapsids 801 are not necessary for alphavirus budding. The EMBO Journal 15, 6495-6505. 
802 Forsell, K., Xing, L., Kozlovska, T., Cheng, R.H., and Garoff, H. (2000). Membrane 803 proteins organize a symmetrical virus. EMBO J. 19, 5081-5091.

804 Fox, J.M., Long, F., Edeling, M.A., Lin, H., van Duijl-Richter, M.K.S., Fong, R.H., Kahle, 805 K.M., Smit, J.M., Jin, J., Simmons, G., et al. (2015). Broadly Neutralizing Alphavirus 806 Antibodies Bind an Epitope on E2 and Inhibit Entry and Egress. Cell 163, 1095-1107.

807 Garoff, H., Sjöberg, M., and Cheng, R.H. (2004). Budding of alphaviruses. Virus Res. $808106,103-116$.

809 de Haan, C.A., Kuo, L., Masters, P.S., Vennema, H., and Rottier, P.J. (1998).

810 Coronavirus particle assembly: primary structure requirements of the membrane 811 protein. J. Virol. 72, 6838-6850.

812 Heilingloh, C.S., and Krawczyk, A. (2017). Role of L-Particles during Herpes Simplex 813 Virus Infection. Front. Microbiol. 8, 2565.

814 Jin, J., Liss, N.M., Chen, D.-H., Liao, M., Fox, J.M., Shimak, R.M., Fong, R.H., Chafets, 815 D., Bakkour, S., Keating, S., et al. (2015). Neutralizing Monoclonal Antibodies Block 816 Chikungunya Virus Entry and Release by Targeting an Epitope Critical to Viral 817 Pathogenesis. Cell Reports 13, 2553-2564.

818 Jin, J., Galaz-Montoya, J.G., Sherman, M.B., Sun, S.Y., Goldsmith, C.S., O’Toole, E.T., 819 Ackerman, L., Carlson, L.-A., Weaver, S.C., Chiu, W., et al. (2018). Neutralizing 820 Antibodies Inhibit Chikungunya Virus Budding at the Plasma Membrane. Cell Host 821 Microbe 24, 417-428.e5.

822 Kail, M., Hollinshead, M., Ansorge, W., Pepperkok, R., Frank, R., Griffiths, G., and 823 Vaux, D. (1991). The cytoplasmic domain of alphavirus E2 glycoprotein contains a short 824 linear recognition signal required for viral budding. EMBO J. 10, 2343-2351.

825 Kim, A.S., Kafai, N.M., Winkler, E.S., Gilliland, T.C., Jr, Cottle, E.L., Earnest, J.T., 826 Jethva, P.N., Kaplonek, P., Shah, A.P., Fong, R.H., et al. (2021). Pan-protective anti827 alphavirus human antibodies target a conserved E1 protein epitope. Cell 184, 4414828 4429.e19. 
829 Kononchik, J.P., Jr, Nelson, S., Hernandez, R., and Brown, D.T. (2009). Helical virus 830 particles formed from morphological subunits of a membrane containing icosahedral 831 virus. Virology 385, 285-293.

832 Kremer, J.R., Mastronarde, D.N., and Mclntosh, J.R. (1996). Computer visualization of 833 three-dimensional image data using IMOD. J. Struct. Biol. 116, 71-76.

834 Laakkonen, P., Auvinen, P., Kujala, P., and Kääriäinen, L. (1998). Alphavirus replicase 835 protein NSP1 induces filopodia and rearrangement of actin filaments. J. Virol. 72, 836 10265-10269.

837 Lamb, K., Lokesh, G.L., Sherman, M., and Watowich, S. (2010). Structure of a 838 Venezuelan equine encephalitis virus assembly intermediate isolated from infected 839 cells. Virology 406, 261-269.

840 Martinez, M.G., Snapp, E.-L., Perumal, G.S., Macaluso, F.P., and Kielian, M. (2014).

841 Imaging the alphavirus exit pathway. J. Virol. 88, 6922-6933.

842 Mastronarde, D.N. (2003). SerialEM: A Program for Automated Tilt Series Acquisition 843 on Tecnai Microscopes Using Prediction of Specimen Position. Microsc. Microanal. 9, 844 1182-1183.

845 Mastronarde, D.N. (2005). Automated electron microscope tomography using robust 846 prediction of specimen movements. J. Struct. Biol. 152, 36-51.

847 Mukhopadhyay, S., Chipman, P.R., Hong, E.M., Kuhn, R.J., and Rossmann, M.G. 848 (2002). In vitro-assembled alphavirus core-like particles maintain a structure similar to 849 that of nucleocapsid cores in mature virus. J. Virol. 76, 11128-11132.

850 Nicola, A.V., Chen, W., and Helenius, A. (1999). Co-translational folding of an 851 alphavirus capsid protein in the cytosol of living cells. Nat. Cell Biol. 1, 341-345.

852 Nikolic, D.S., Lehmann, M., Felts, R., Garcia, E., Blanchet, F.P., Subramaniam, S., and 853 Piguet, V. (2011). HIV-1 activates Cdc42 and induces membrane extensions in 854 immature dendritic cells to facilitate cell-to-cell virus propagation. Blood 118, 4841- 
856 Noranate, N., Takeda, N., Chetanachan, P., Sittisaman, P., A-Nuegoonpipat, A., and 857 Anantapreecha, S. (2014). Characterization of chikungunya virus-like particles. PLoS 858 One 9, e108169.

859 Pavan, A., Lotti, L.V., Torrisi, M.R., Migliaccio, G., and Bonatti, S. (1987). Regional 860 distribution of Sindbis virus glycoproteins on the plasma membrane of infected baby 861 hamster kidney cells. Exp. Cell Res. 168, 53-62.

862 Pettersen, E.F., Goddard, T.D., and Huang, C.C. (2004). UCSF Chimera-a 863 visualization system for exploratory research and analysis. Journal of.

864 Pettersen, E.F., Goddard, T.D., Huang, C.C., Meng, E.C., Couch, G.S., Croll, T.I., 865 Morris, J.H., and Ferrin, T.E. (2021). UCSF ChimeraX: Structure visualization for 866 researchers, educators, and developers. Protein Sci. 30, 70-82.

867 Rigort, A., Bäuerlein, F.J.B., Villa, E., Eibauer, M., Laugks, T., Baumeister, W., and 868 Plitzko, J.M. (2012). Focused ion beam micromachining of eukaryotic cells for 869 cryoelectron tomography. Proc. Natl. Acad. Sci. U. S. A. 109, 4449-4454.

870 Ruiz-Guillen, M., Gabev, E., Quetglas, J.I., Casales, E., Ballesteros-Briones, M.C., 871 Poutou, J., Aranda, A., Martisova, E., Bezunartea, J., Ondiviela, M., et al. (2016).

872 Capsid-deficient alphaviruses generate propagative infectious microvesicles at the 873 plasma membrane. Cell. Mol. Life Sci. 73, 3897-3916.

874 Sanjuán, R. (2021). The Social Life of Viruses. Annu Rev Virol.

875 Selvarajah, S., Sexton, N.R., Kahle, K.M., Fong, R.H., Mattia, K.-A., Gardner, J., Lu, K., 876 Liss, N.M., Salvador, B., Tucker, D.F., et al. (2013). A neutralizing monoclonal antibody 877 targeting the acid-sensitive region in chikungunya virus E2 protects from disease. PLoS 878 Negl. Trop. Dis. 7, e2423.

879 Sherer, N.M., and Mothes, W. (2008). Cytonemes and tunneling nanotubules in cell-cell 880 communication and viral pathogenesis. Trends in Cell Biology 18, 414-420. 
Silva, L.A., and Dermody, T.S. (2017). Chikungunya virus: epidemiology, replication, disease mechanisms, and prospective intervention strategies. J. Clin. Invest. 127, 737749.

884 Snyder, J.E., Azizgolshani, O., Wu, B., He, Y., Lee, A.C., Jose, J., Suter, D.M., Knobler, 885 C.M., Gelbart, W.M., and Kuhn, R.J. (2011). Rescue of infectious particles from preassembled alphavirus nucleocapsid cores. J. Virol. 85, 5773-5781.

887 Soonsawad, P., Xing, L., Milla, E., Espinoza, J.M., Kawano, M., Marko, M., Hsieh, C., 888 Furukawa, H., Kawasaki, M., Weerachatyanukul, W., et al. (2010). Structural evidence 889 of glycoprotein assembly in cellular membrane compartments prior to Alphavirus 890 budding. J. Virol. 84, 11145-11151.

891 Sowinski, S., Jolly, C., Berninghausen, O., Purbhoo, M.A., Chauveau, A., Köhler, K., 892 Oddos, S., Eissmann, P., Brodsky, F.M., Hopkins, C., et al. (2008). Membrane 893 nanotubes physically connect T cells over long distances presenting a novel route for 894 HIV-1 transmission. Nat. Cell Biol. 10, 211-219.

895 Stange, A., Lüftenegger, D., Reh, J., Weissenhorn, W., and Lindemann, D. (2008). Subviral particle release determinants of prototype foamy virus. J. Virol. 82, 9858-9869.

897 Sun, S., Xiang, Y., Akahata, W., Holdaway, H., Pal, P., Zhang, X., Diamond, M.S., 898 Nabel, G.J., and Rossmann, M.G. (2013). Structural analyses at pseudo atomic 899 resolution of Chikungunya virus and antibodies show mechanisms of neutralization. 900 Elife 2, e00435.

901 Suomalainen, M., Liljeström, P., and Garoff, H. (1992). Spike protein-nucleocapsid 902 interactions drive the budding of alphaviruses. J. Virol. 66, 4737-4747.

903 Sutton, G., Sun, D., Fu, X., Kotecha, A., Hecksel, C.W., Clare, D.K., Zhang, P., Stuart, 904 D.I., and Boyce, M. (2020). Assembly intermediates of orthoreovirus captured in the 905 cell. Nat. Commun. 11, 4445.

906 Tan, T.Y., Fibriansah, G., and Lok, S.-M. (2020). Capsid protein is central to the birth of 907 flavivirus particles. PLoS Pathog. 16, e1008542. 
908 Tang, J., Jose, J., Chipman, P., Zhang, W., Kuhn, R.J., and Baker, T.S. (2011).

909 Molecular links between the E2 envelope glycoprotein and nucleocapsid core in Sindbis 910 virus. J. Mol. Biol. 414, 442-459.

911 Taylor, G.M., Hanson, P.I., and Kielian, M. (2007). Ubiquitin depletion and dominant-

912 negative VPS4 inhibit rhabdovirus budding without affecting alphavirus budding. J. Virol.

$91381,13631-13639$.

914 Vaillant, A. (2021). HBsAg, Subviral Particles, and Their Clearance in Establishing a 915 Functional Cure of Chronic Hepatitis B Virus Infection. ACS Infect Dis 7, 1351-1368.

916 Vignuzzi, M., and López, C.B. (2019). Defective viral genomes are key drivers of the 917 virus-host interaction. Nat Microbiol 4, 1075-1087.

918 Vijayakrishnan, S., McElwee, M., Loney, C., Rixon, F., and Bhella, D. (2020). In situ 919 structure of virus capsids within cell nuclei by correlative light and cryo-electron 920 tomography. Sci. Rep. 10, 17596.

921 Wan, J.J., Brown, R.S., and Kielian, M. (2020). Berberine Chloride is an Alphavirus 922 Inhibitor That Targets Nucleocapsid Assembly. MBio 11.

923 Wang, J.C.-Y., Chen, C., Rayaprolu, V., Mukhopadhyay, S., and Zlotnick, A. (2015).

924 Self-Assembly of an Alphavirus Core-like Particle Is Distinguished by Strong

925 Intersubunit Association Energy and Structural Defects. ACS Nano 9, 8898-8906.

926 Wang, P.-G., Kudelko, M., Lo, J., Siu, L.Y.L., Kwok, K.T.H., Sachse, M., Nicholls, J.M., 927 Bruzzone, R., Altmeyer, R.M., and Nal, B. (2009). Efficient Assembly and Secretion of 928 Recombinant Subviral Particles of the Four Dengue Serotypes Using Native prM and E 929 Proteins. PLoS ONE 4, e8325.

930 Williamson, L.E., Reeder, K.M., Bailey, K., Tran, M.H., Roy, V., Fouch, M.E., Kose, N., 931 Trivette, A., Nargi, R.S., Winkler, E.S., et al. (2021). Therapeutic alphavirus cross932 reactive E1 human antibodies inhibit viral egress. Cell 184, 4430-4446.e22.

933 Yan, N., and Chen, Z.J. (2012). Intrinsic antiviral immunity. Nature Immunology 13, 
$934 \quad 214-222$.

935 Zhang, R., Hryc, C.F., Cong, Y., Liu, X., Jakana, J., Gorchakov, R., Baker, M.L.,

936 Weaver, S.C., and Chiu, W. (2011). 4.4 A cryo-EM structure of an enveloped alphavirus

937 Venezuelan equine encephalitis virus. EMBO J. 30, 3854-3863.

938 Zheng, S.Q., Palovcak, E., Armache, J.-P., Verba, K.A., Cheng, Y., and Agard, D.A.

939 (2017). MotionCor2: anisotropic correction of beam-induced motion for improved cryo-

940 electron microscopy. Nat. Methods 14, 331-332. 


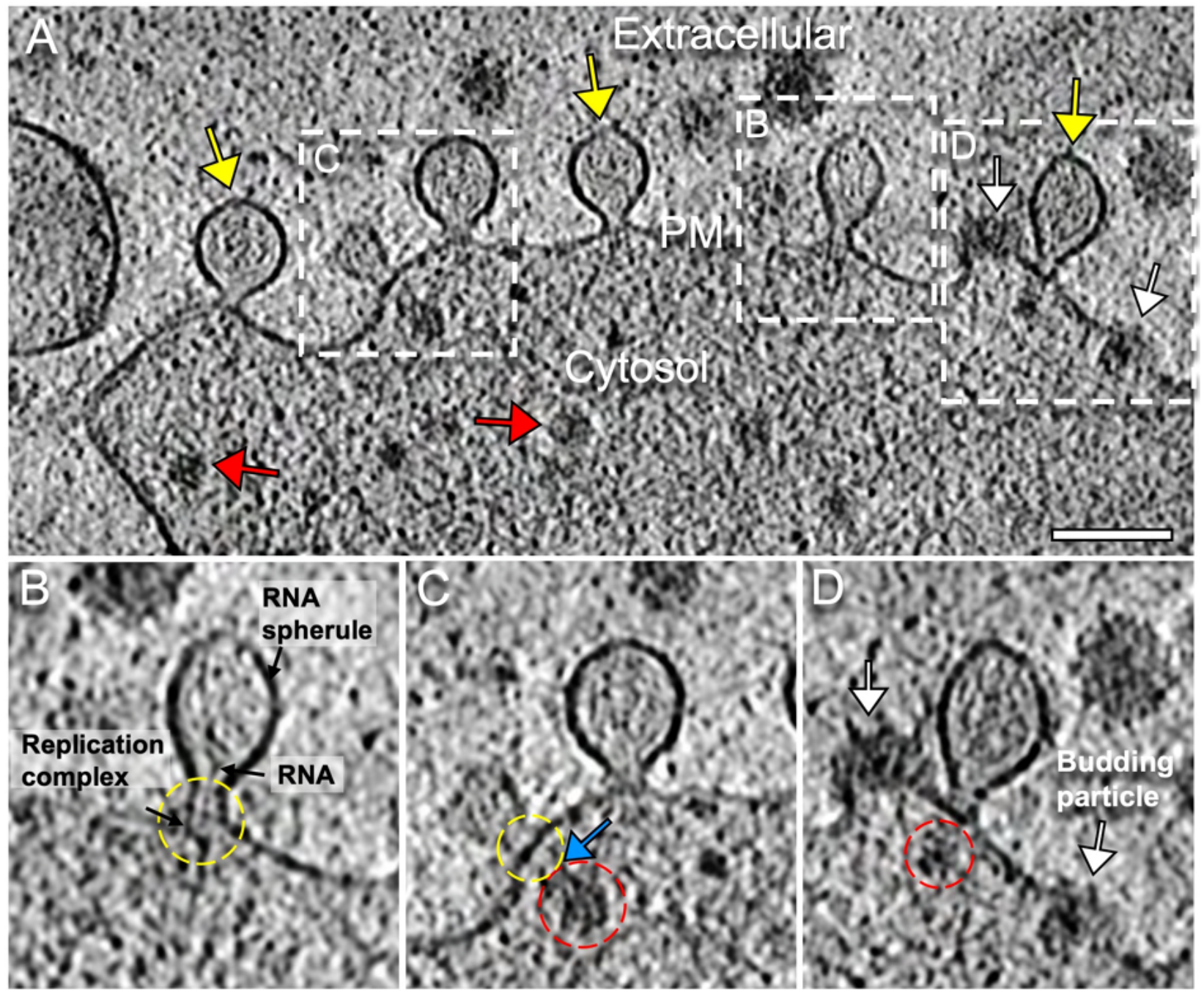

Fig. S1. RNA replication spherules on the cell surface.

(A) Tomogram slice displaying cell periphery with RNA replication spherules (yellow arrows), budding intermediates (white arrows) and apparently cytosolic NLPs (red arrows). Scale bar: $100 \mathrm{~nm}$. (B) Enlarged tomogram slice of RNA replication spherule with components labeled. Proposed location of the replication complex at the neck of spherules was indicated with a yellow dashed circle. (C) Enlarged slice view of a RNA spherule with a NLP (red dashed circle) in close proximity to the spherule neck (yellow dashed circle), with some thin density (blue arrow) connecting in between. (D) Enlarged slice view of RNA spherule in close proximity to another apparently incompletelyassembled NLP (red dashed circle) and multiple budding particles (white arrows). 


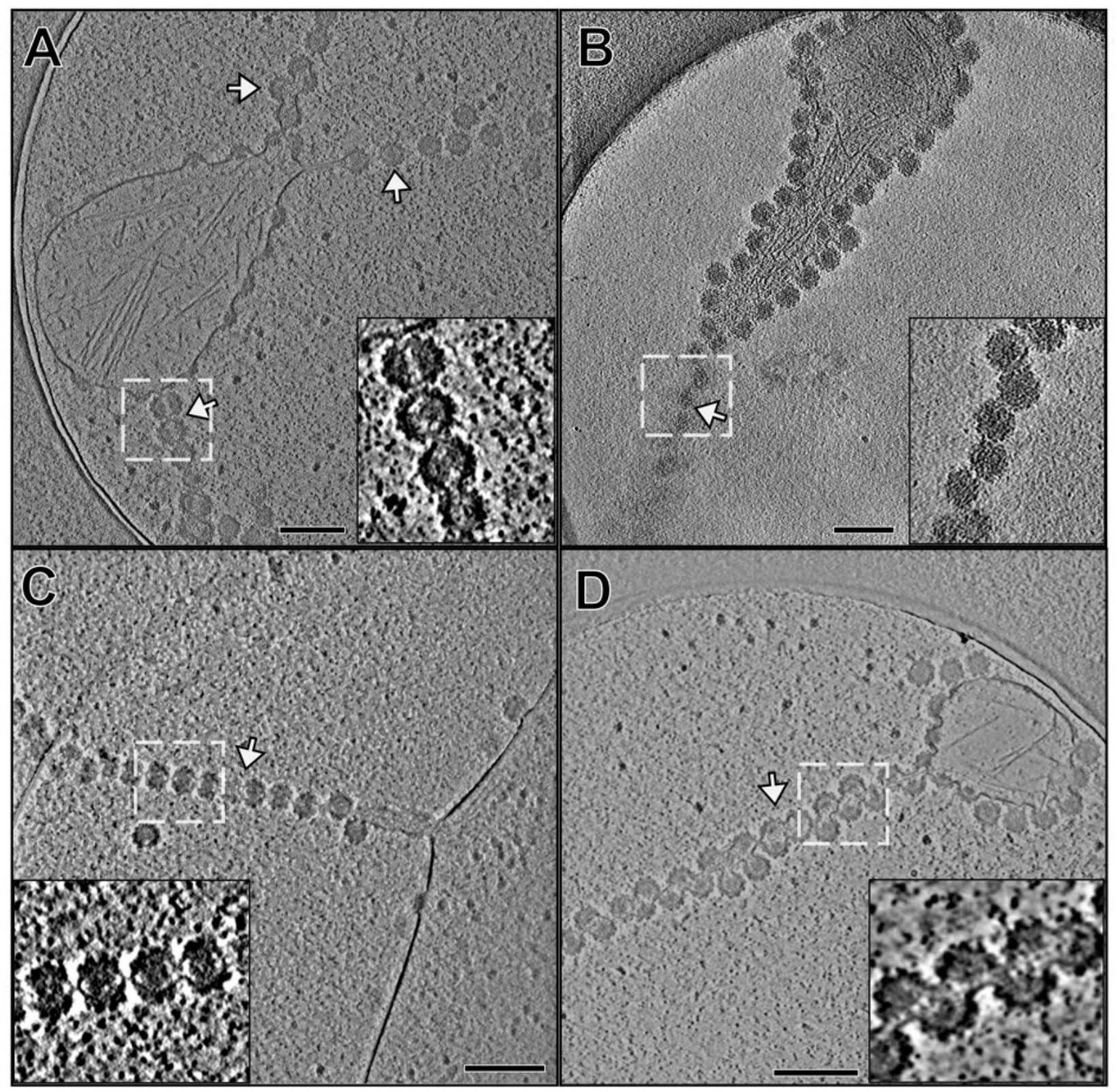

Fig. S2. Strands of incomplete particles extend from the cell surface.

(A-D) Tomogram slice images display thin extensions of virus budding intermediates in multiple conformations (white dashed boxes, inset images). In all observed cases, beads of linked particles form at the convergences of two membrane surfaces with near-continuous budding intermediates. Scale bars: $200 \mathrm{~nm}$. 
A

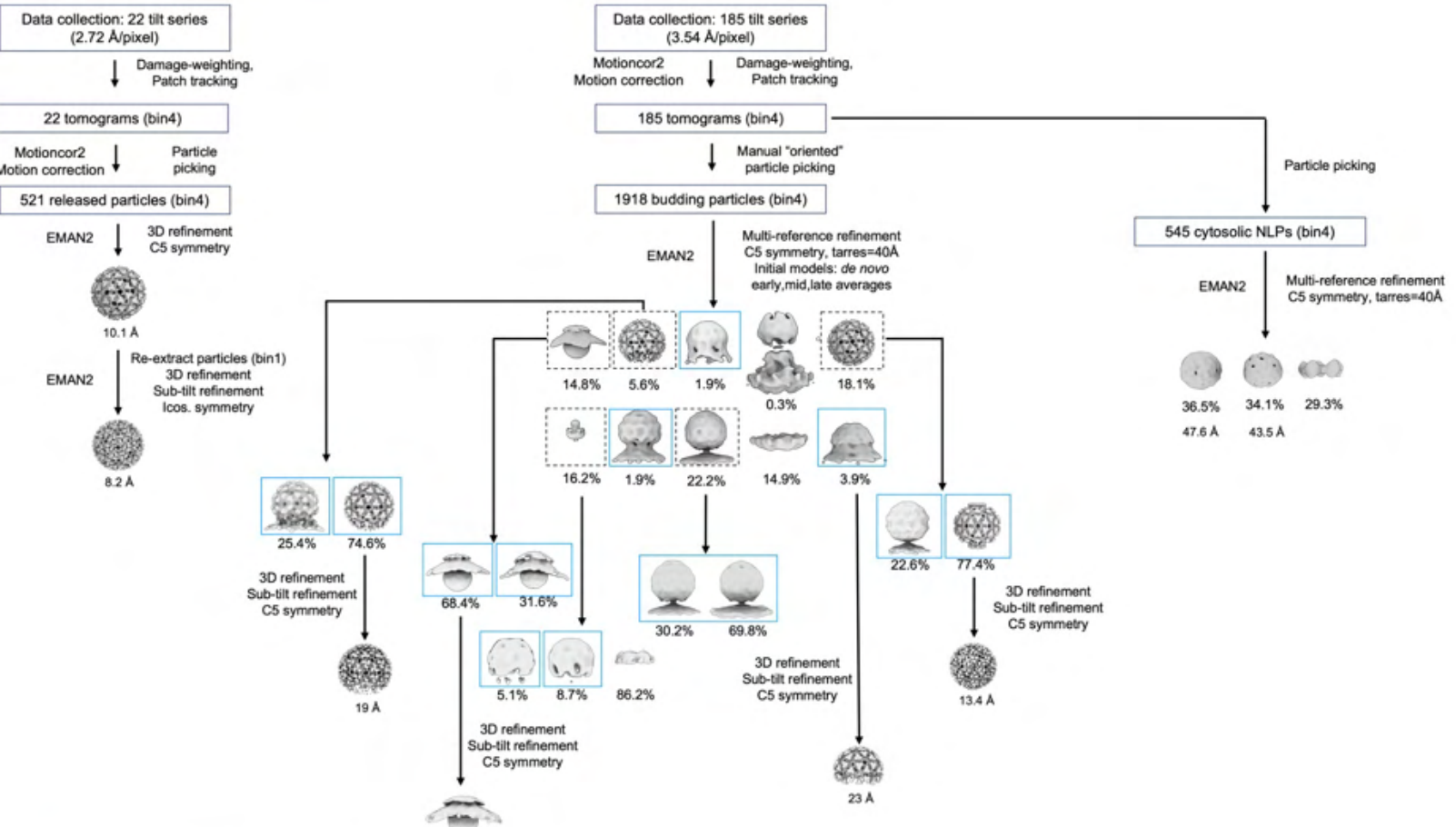

$43.7 \mathrm{~A}$

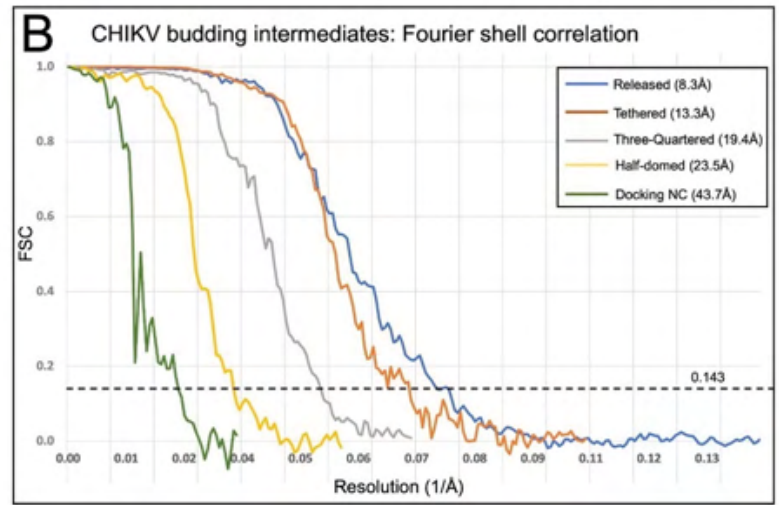

C

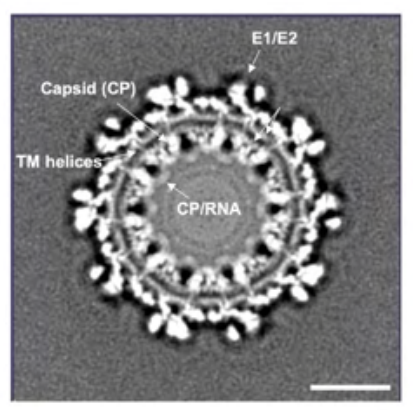

D

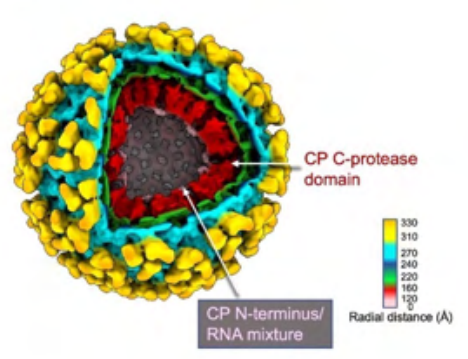

Fig. S3. CryoET data processing workflow and resolutions of STA structures. (A) CryoET and data processing workflow. For budding intermediates subvolume classification (middle), particles in 5 of the 10 classes (dashed black boxes) from the first multi-reference refinement were subjected to additional classification. 12 distinct intermediate budding conformations (blue boxes) were resolved after two rounds of classification. Note: two maps displayed within a single blue box merged into one class due to overall structure similarity. (B) Gold standard Fourier shell correlation (FSC) plots of subtomogram average structures of budding intermediates and released virions. (C) Slice view of $3 \mathrm{D}$ reconstruction of released CHIKV virions. Subnanometer $(8.3 \AA)$ resolution of structure is evident by resolved TM helices of E1/E2 in the lipid bilayer. (D) Radial-colored density map of icosahedral CHIKV particle reveals density of CP Cprotease domain (red) as well as an ordered density layer below (pink) that is possibly CP N-terminus+gRNA. 

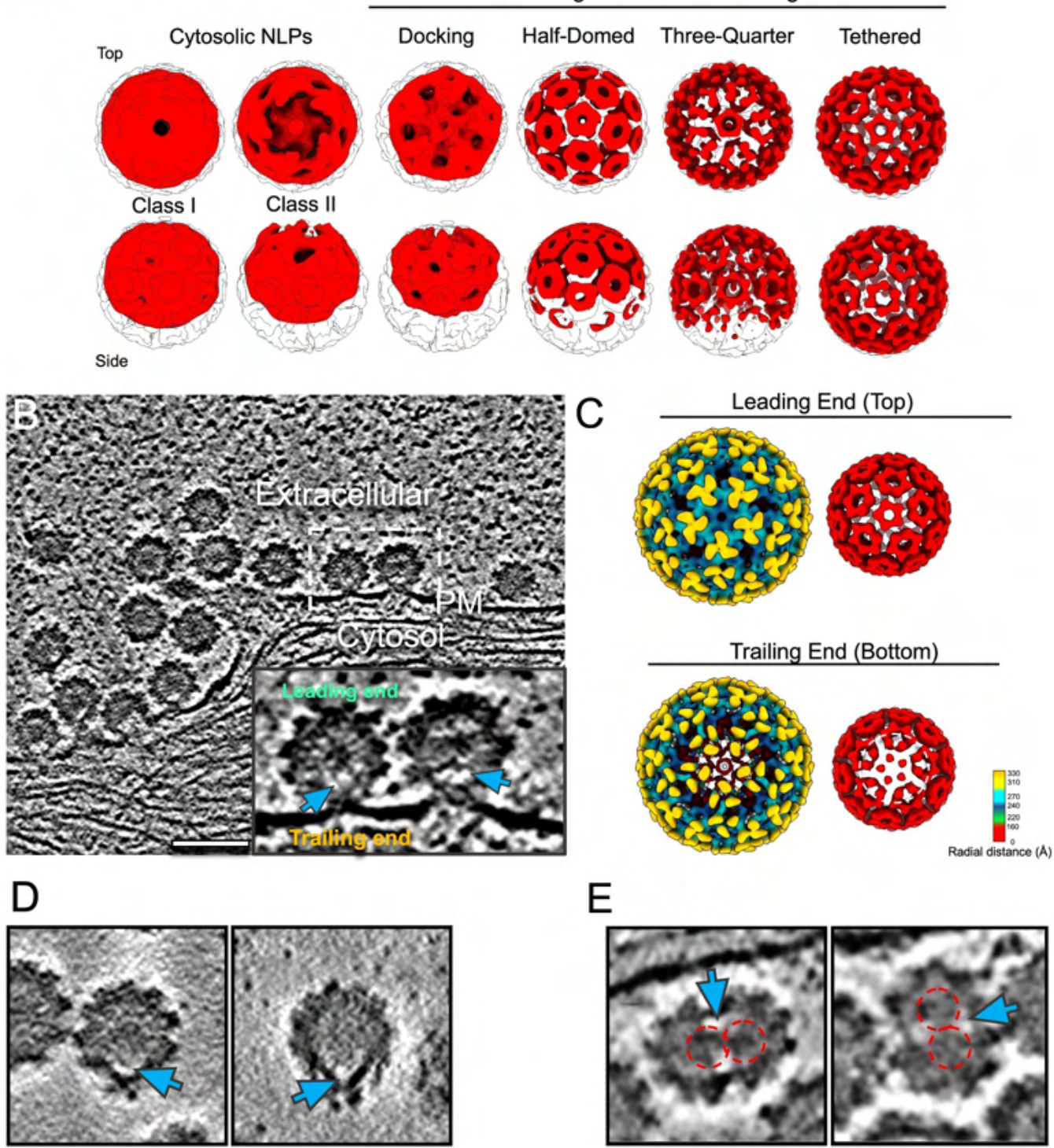

E
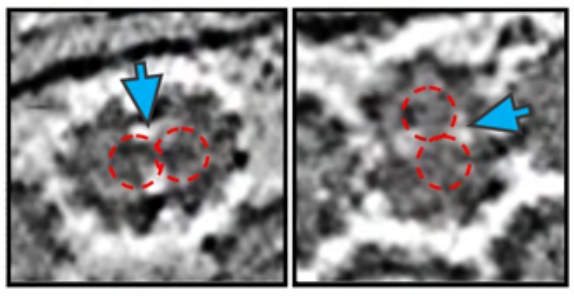

Fig. S4. Non-icosahedral features of virus particles.

(A) Subtomogram average structures of cytosolic NLPs and NCs from buddingintermediate 3D reconstructions (shown in red). NC structures are overlaid on the density map of the NC from released CHIKV virion (white, transparent). (B) Volta phase plate tomogram slice images of PM with multiple budding intermediates, including late-stage ("tethered") particles. Inset is the zoom-in view of the boxed area in (B) displaying relatively absent density at the base of late-stage budding particles (blue arrow). Top of a budding particle, furthest from PM is defined as the leading end, while base of the particle is defined as the trailing end. (C) Subtomogram average structure of "tethered intermediate" shows icosahedral symmetry at the leading end, while trailing end of average shows a disordered final penton and distorted capsomer density in those hexamer units below. Relatively absent density between NC and viral envelope (blue arrows) was also observed at (D) the nonspherical pole of released virions, and $(E)$ the released multi-cored particles. NCs in multi-core particles are labeled with dashed red circles. 


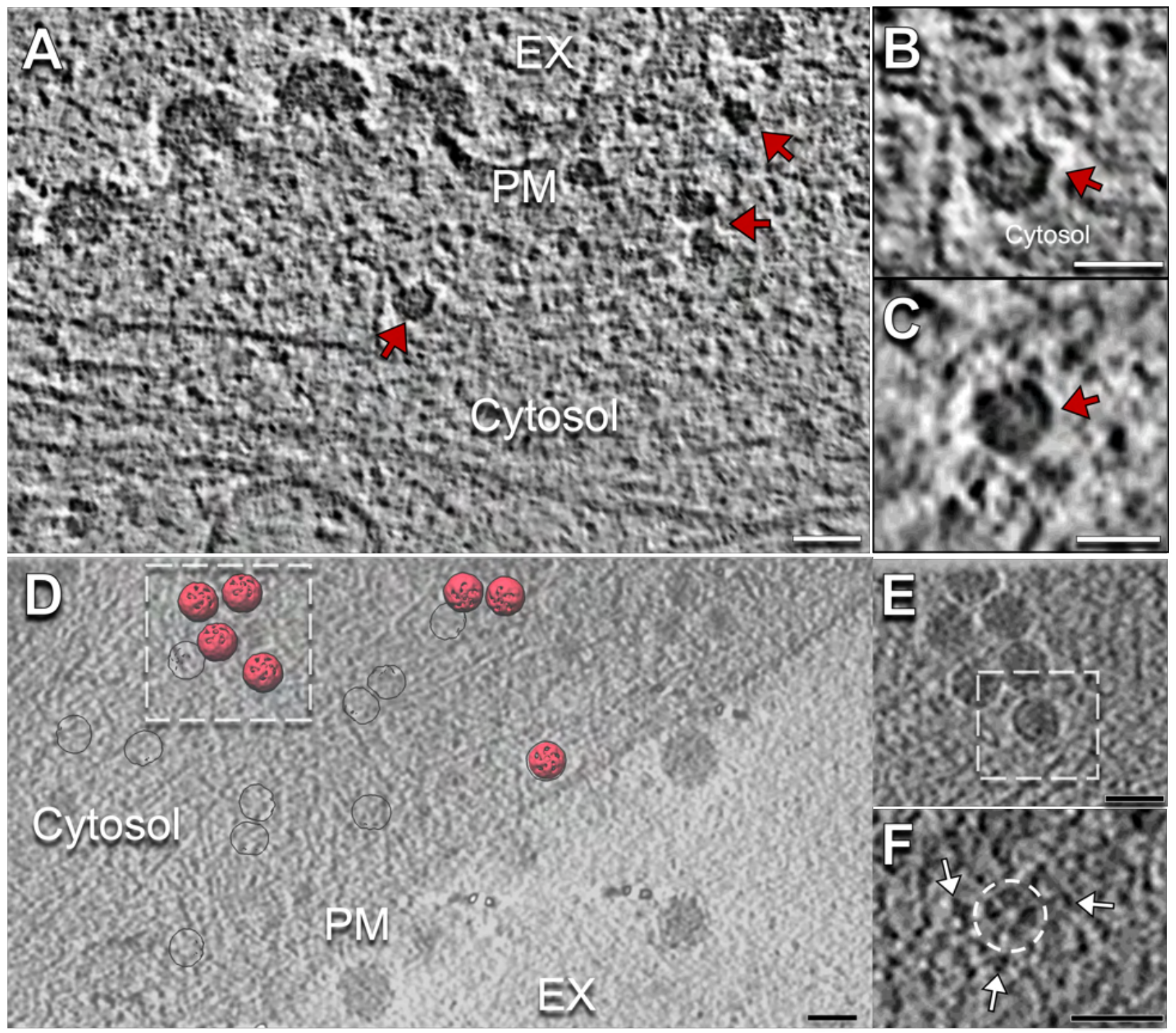

Fig. S5. Orientation of cytosolic NLPs.

(A) Volta-phase plate tomogram slice image shows budding cell periphery with apparently cytosolic NLPs (red arrows). Scale bar: $50 \mathrm{~nm}$. (B,C) Zoom-in views of NLPs (red arrows). Scale bars: $30 \mathrm{~nm}$. (D) Tomogram slice image of cell with subvolume averages of NLP class II (red, Fig. S4) mapped back to the tomogram based on the refined orientation of each particle. 5-fold density consistently oriented towards the PM surface in slices above. (E) Tomogram slice image displays cluster of NLPs (red arrow). Scale bar: 50 $\mathrm{nm}$.(F) In a tomogram slice directly above a single NLP (white box), a penton of spikes (dashed white circle) is identified along with nearby spikes (white arrows). Scale bar: 50nm. 


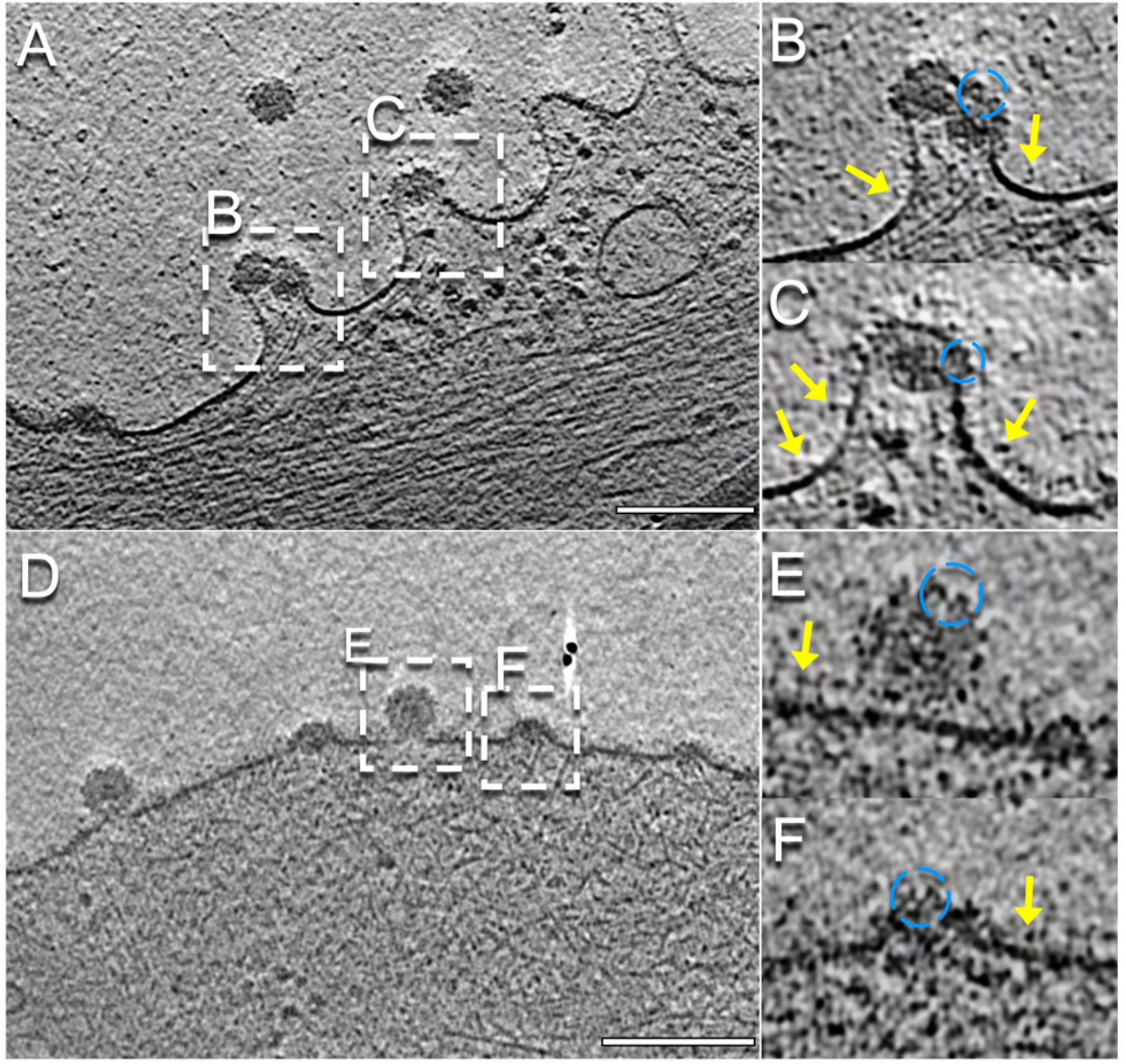

Fig. S6. Different conformations of spikes before and after assembly in budding shells.

$(A, D)$ Tomogram slice displaying cell periphery with budding intermediates. Scale bars: $200 \mathrm{~nm}$. (B, C, E, F) Enlarged tomogram slices of budding particles. Spike side-views on budding intermediates surface (blue dashed circle) displayed characteristic conformation of spikes assembled in lattice, in contrast to potential individual spikes (yellow arrows) near the base of budding intermediates. 


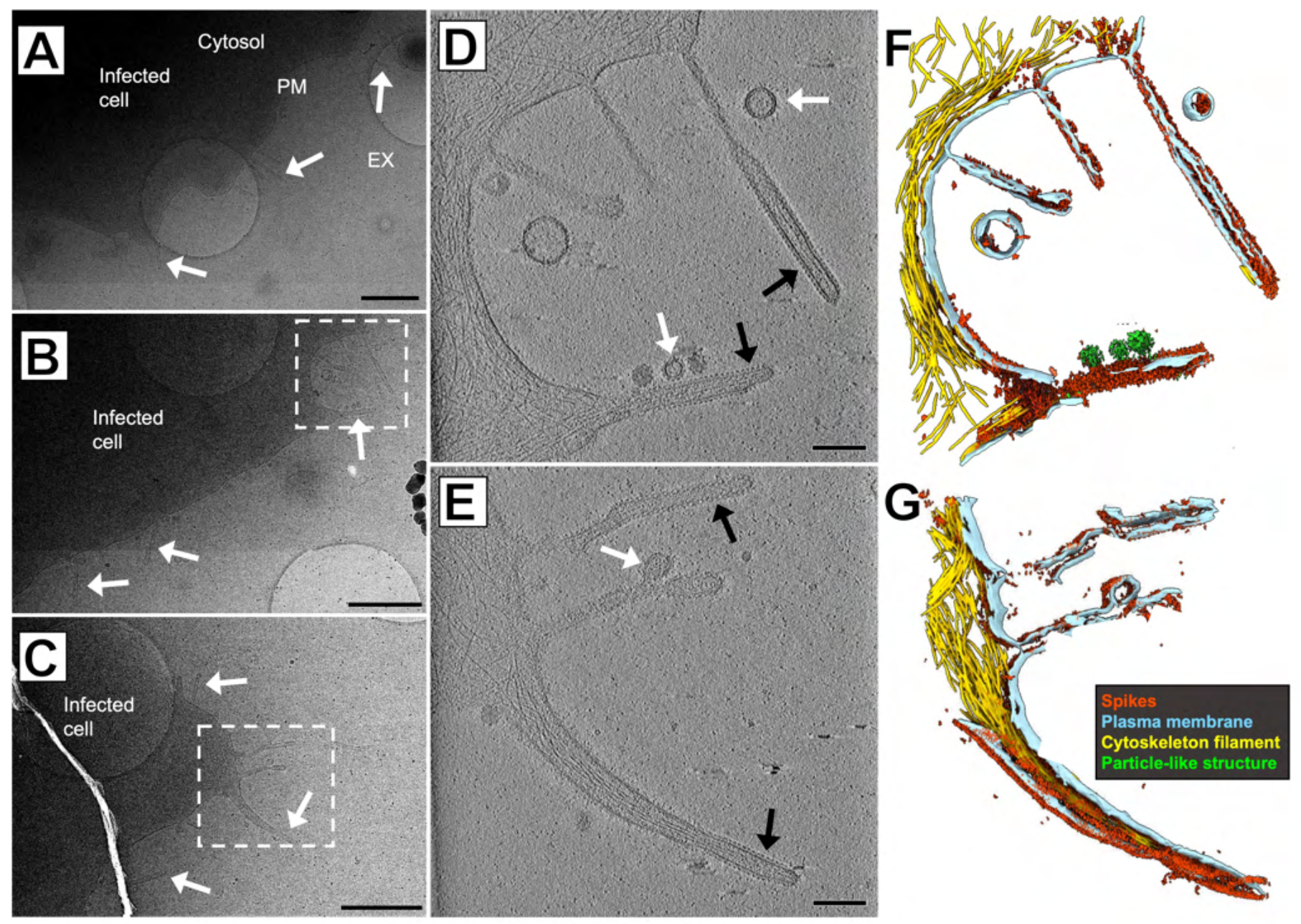

Fig. S7. Self-assembled structures of spikes alone.

(A-C) Images of CHIKV-infected cell peripheries display cell extensions (white arrows) directly from the cell body. Scale bars: $1 \mu \mathrm{m}$. ( $D \& E$ ) Zoom-in images of the boxed regions in $B \& C$, display tubular protein arrays (black arrows) at the terminal end and released vesicle-like assembly products lacking dense cores (white arrows). Scale bars: $200 \mathrm{~nm}$. (F \& G) 3D segmentations of cellular features corresponding to ( $D \& E$ ) show the surfaces of tubular extensions contain dense spikes. Segmentations reveal spikes on the surface of tubular membrane extensions that largely exclude bundled cytoskeleton filaments. 
Data collection and processing

Docking Half-domed Three-Quartered Tethered Released

Magnification

Voltage (kV)

$\begin{array}{ccc}39,000 & 39,000 & 39,000 \\ 200 & 200 & 200 \\ 90 & 90 & 90 \\ -3 \text { to }-5.5 & -3 \text { to }-5.5 & -3 \text { to }-5.5 \\ 3.54 & 3.54 & 3.54 \\ \text { C5 } & \text { C5 } & \text { C5 } \\ 197 & 322 & 172 \\ 43.7 & 23.5 & 19.4\end{array}$

$\begin{array}{cc}39,000 & 53,000 \\ 200 & 300 \\ 90 & 120 \\ -3 \text { to }-5.5 & -2 \text { to }-4 \\ 3.54 & 2.72 \\ \text { C5 } & \text { Icos } \\ 364 & 567 \\ 13.3 & 8.3\end{array}$

Table S1. Cryo-ET data analysis and subtomogram averaging statistics. 
Thin extension, neighboring budding particles with NCs

Thin extension, spike array, no neighboring NCs

Note: diameter of virion approx. $70 \mathrm{~nm}$

Table S2. Average diameters of helical tubes formed by hexagonal spike arrays in different cellular contexts. 